\title{
Die euripideische Tragödie auf der Bühne der Antike $^{1}$
}

\begin{abstract}
This article attempts to outline the reception of Euripides' tragedies after the poet's death in 407/6 BCE. The reception can be described as a process of specialization. In its first phase (until the end of the $4^{\text {th }}$ cent. BCE) the production on stage remains the dominant form in which Athenians encountered the plays of Euripides. Only at the end of the $4^{\text {th }}$ century (marked by Aristotle's Poetics) did Euripides also become a 'textual phenomenon': from then onwards a differentiation between 'theater' and 'book' developed, partly bridged by the hybrid form of recitation of Euripides' plays. In the Imperial period, festivals offered numerous occasions for producing plays, but the testimonies of production show that mostly only spectacular scenes from Euripidean drama were performed.
\end{abstract}

Als Euripides im attischen Jahr 407/6 starb, konnte man dies - jedenfalls nach Ausweis der Frösche des Aristophanes - als eine Zäsur der athenischen Theatergeschichte begreifen. Kein Wunder, hatte doch seit seiner ersten Beteiligung am Agon 455 Euripides kontinuierlich Tetralogien zu den Dionysien oder wenigstens Dilogien zu den Lenäen beigesteuert. Und auch wenn er hierbei nur viermal zu Lebzeiten (zuerst 441, ferner 428, die beiden anderen Siege lassen sich nicht lokalisieren) den Sieg errungen hatte, ${ }^{2}$ so kann man aus der rekonstruierbaren Zahl von insgesamt 92 Stücken folgern, die er verfasst hat (78 hiervon waren den alexandrinischen Philologen verfügbar), ${ }^{3}$ dass er an wohl mehr als 20 Dionysien beteiligt war, hierunter nachweislich in den Jahren 455, 441, 438, 431, 428, 415, 412 und 408. Nach seinem Tod wurde eine weitere Trilogie - Bakchen, Aulische Iphigenie und Alkmaion - wohl von seinem gleichnamigen Sohn ${ }^{4}$ auf die Bühne gebracht, die einen weiteren Sieg erzielte.

Schwieriger ist es, die Präsenz des Euripides auf den außerathenischen Bühnen, sei es in den attischen Demen, sei es in anderen Städten Griechenlands zu seinen

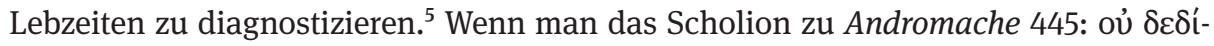

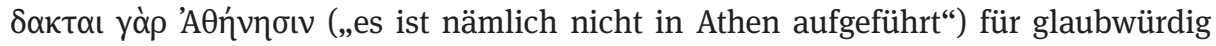
hält, wäre diese Tragödie nicht in Athen aufgeführt worden. Ein weiterer Kandidat ist der Archelaos, wohl zwischen 408 und dem Tod des Dichters verfasst, der mit einer

\footnotetext{
1 Für Hinweise und Kritik danke ich Dr. Annamaria Peri. Soweit nicht anders ausgewiesen stammen die deutschen Übersetzungen von mir.

2 Siehe TrGF 5, T 65a.

3 Hierzu insgesamt Kannicht 1996 und unten in diesem Band der Beitrag von Rosa Maria Piccione.

4 Vita Euripidis IA, TrGF 5, T 1,8.

5 Vgl. hierzu auch Easterling 1994.
}

https://doi.org/10.1515/9783110677072-003 
gewissen Wahrscheinlichkeit in Makedonien für den gleichnamigen König auf die Bühne kam. ${ }^{6}$ Dass das spezifische Interesse gerade an diesen beiden Stücken auf Bühnen außerhalb Athens in - wie auch immer gelagerten - (kultur-)politischen Dimensionen gelegen haben könnte und nicht den Dichter Euripides und seine Kunst an sich betraf, wird man mindestens erwägen dürfen.

\section{Euripides im 4. Jh. v. Chr.: Wiederaufführungen und literarische Rezeption}

\subsection{Das Forschungsnarrativ und seine Probleme}

Es gehört zu den Topoi der griechischen Literaturgeschichte, dass Euripides nach seinem Tod beliebter und erfolgreicher war als zu Lebzeiten. ${ }^{7}$ Und in der Tat dürfte Euripides nach Homer der am häufigsten und deswegen auch am intensivsten rezipierte griechische Dichter der griechischen Kultur bis in die Spätantike sein. Doch wie kommt es dazu? Die Forschung neigt seit langem zu folgendem Narrativ: ${ }^{8}$ Die Formen der intensiven Euripides-Rezeption seien bereits am Ende des 5. Jh. v. Chr. vorgegeben. Denn neben der Gegenwart euripideischer Stücke auf den Theaterbühnen (ich benutze diesen Terminus hier prägnant) seien sie auch als Texte greifbar. Symptomatisch erscheint hierfür dann bereits das Leseerlebnis, das 405 der aristophanische Theatergott Dionysos in den Fröschen artikuliert:

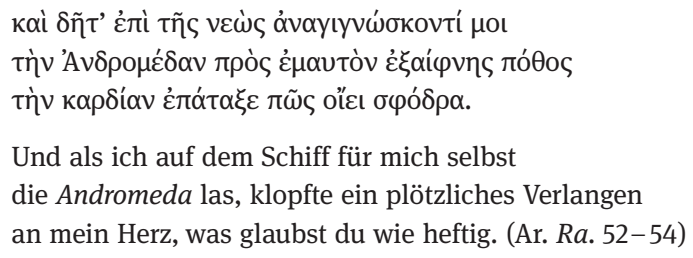

Es war also eine Individual-Lektüre einzelner Stücke möglich, und die reichen Proben aus euripideischen Stücken, die Aristophanes in den Fröschen metrischer oder dramentechnischer Analyse aussetzt, legen bereits Sammlungen von Stücken nahe. ${ }^{9}$

6 Siehe hierzu die Diskussion von Harder 1985, 125-127.

7 Vgl. etwa Xanthakis-Karamanos 1980, $28-34$ zum 4. Jh. v.Chr.

8 Eine neuere Behandlung der Euripides-Rezeption in der Antike fehlt: Lauriola u. Demetriou 2015 bieten lediglich Einzeldarstellungen zur Rezeption der einzelnen Stücke insbesondere in der Neuzeit. So bleiben - wegen des Materialreichtums - wichtig Funke 1965/66 und die Übersichtsdarstellung von Schmid 1940, 823-838. Eine Übersicht über die Aufführungsgeschichte der drei kanonischen Tragiker gibt Nervegna 2014.

9 Interessant erscheint hierbei, dass offenbar am Ende des 5. Jh. v.Chr. mehr Stücke als Buch kursierten als später in Alexandria gesammelt werden konnten. So parodieren Aristophanes' Frösche 
Diese Beliebtheit des Euripides brachte also nicht nur den aristophanischen Theatergott Dionysos dazu, in den Hades hinabzusteigen, um Euripides zurückzuholen, sondern sei, so die Tendenz der Forschung, auch einer der Faktoren gewesen sein, der eine tiefgreifende Änderung der Dionysien hervorrief: Für das Jahr des Archons Theodotos (387/6) teilt die berühmte, als „Fasten“ bezeichnete Inschrift IG II 2318, col. VIII, mit: $\pi \alpha \lambda \alpha$ เòv $\delta \rho \tilde{\alpha} \mu \alpha \pi \rho \tilde{\omega} \tau o[v] \pi \alpha \rho \varepsilon \delta i ́ \delta \alpha \xi \alpha \nu$ oi $\tau \rho \alpha y[\omega เ \delta o i ́]$ (,ein altes Stück führen nebenher auf die Tragöden“). Augenscheinlich getragen von den Tragödienschauspielern ${ }^{10}$ erfolgte die Wiederaufführung einer ,alten ${ }^{6}$ Tragödie als Beiprogramm an den Dionysien. ${ }^{11}$ Von nun an seien die euripideischen Dramen fester wie beliebter Bestandteil des Repertoire-Theaters, und ihre Verbreitung als Lesetexte sicherte ihren Einfluss in der griechischen Kultur, verstärkt durch die Sammelarbeit und editorisch-kommentatorischen Bemühungen der alexandrinischen Philologen, bis in die byzantinische Zeit.

So etwa das Narrativ. Genauere Betrachtung des 4. Jh. v. Chr. führt freilich dazu, dass man die für dieses Narrativ wichtigen Bausteine gar nicht so einfach auffinden kann. Zunächst: In diesem Jahrhundert wird Euripides gar nicht so intensiv in der Literatur rezipiert, wie man eigentlich erwarten müsste; sodann: auch die Bezeugung von Wiederaufführungen euripideischer Stücke ist nicht so überwältigend belegt, wie erwartbar wäre. ${ }^{12}$ Ich setze also neu an und beginne mit einem auf den ersten Blick irritierenden Zeugnis, einem Lemma aus dem sog. 5. Bekkerschen Lexikon, einer

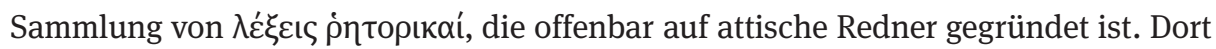

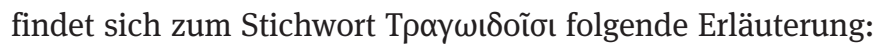

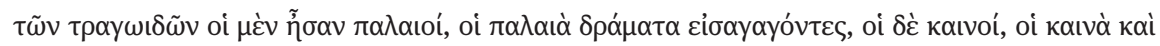

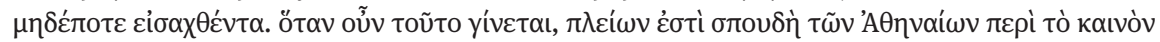

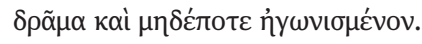

Unter den Tragöden gab es die ,Alten', die alte Dramen auf die Bühne brachten, und die ,Neuen', die neue und noch nicht aufgeführte Stücke spielten. Sooft dies geschieht, gilt der größere Eifer der Athener dem neuen und noch nicht im Wettbewerb gezeigten Drama. ${ }^{13}$

Nimmt man die Information ernst, dass die Athener mit größerem Eifer die neuen und noch nicht im Wettkampf (sc. an den Dionysien) eingesetzten Dramen als die ,alten“ Dramen verfolgten, ${ }^{14}$ so stellen sich einige Fragen: Warum kam es dann zur Wieder-

(1206-1208 bzw. 1400) Euripides-Stücke, die nicht mehr in Alexandria nachgewiesen werden konnten. Siehe dazu Kannicht 1996, 29 bzw. dessen adnotatio in TrGF 5,2 zu F 846 und F 888.

10 Welcker 1841, 1282 hat die immer noch brauchbare Hypothese aufgestellt, mit úंокрıтńs werde der

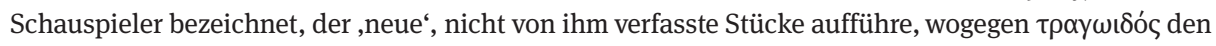
Schauspieler bezeichne, der auf der Basis eigener Auswahl alte Stücke wiederaufführe.

11 Text nach Millis u. Olson 2012; dort (56) auch der Kommentar zu diesem Eintrag in der Inschrift.

12 Vgl. dazu Wagner 1995.

13 Text nach Bekker 1814, 309.

14 Schäfer 1885, 241 Anm. 3 behauptet einen „später geltenden Vorrang“ der Schauspieler alter vor denen neuer Dramen. Sein ,Beleg‘ Welcker 1841, 1278-1280 trägt dies nicht. 
aufführung ,alter' Dramen, zumal dies eine organisatorische Neuerung bedeutete, die insbesondere in einer vormodernen, vergangenheitsorientierten Polis besonderen Aufwands bedurfte, um sich gegen die in den Polis-Strukturen verankerte Beharrungskraft der etablierten Organisationsform durchsetzen zu können. Oder anders formuliert: Das Lemma des Bekkerschen Lexikons legt nahe, dass man in Athen mit den ,neuen' Tragödien sehr zufrieden war, also eigentlich keine Veranlassung zur Veränderung des Jahres 386 bestand. Warum kam sie dennoch zustande? Gab es einen (Leidens-)Druck, der die Neuerung ermöglichte oder gar forderte? Und schließlich: Was bedeutet der besondere Eifer der Athener für neue Stücke für die Wiederaufführung der alten?

Ein zweites Irritament stellt sich ein, wenn man auf die Texte bzw. Text-Corpora des 4. Jh. v.Chr. blickt. Denn Euripides - wie übrigens auch die anderen Tragiker erscheint hier gar nicht so häufig, wie man angesichts der Beliebtheit erwarten müsste, von der unser Forschungsnarrativ ausgeht. Blickt man nämlich darauf, wie oder ob Euripides bei den Autoren dieses Jahrhunderts der Prosa benutzt oder zitiert wird, ergeben sich erstaunliche Lücken.

So finden sich keinerlei Hinweise auf Euripides bei Xenophon, Lysias, Isaios oder Hypereides; ${ }^{15}$ im nicht kleinen Corpus der Schriften des Isokrates fehlt jeder explizite Verweis auf unseren Tragiker. ${ }^{16}$ Dieses ,flächige' Fehlen in zentralen Autoren bzw. zentralen Texten für eine zumindest athenische Öffentlichkeit weist darauf, dass im intellektuellen griechischen bzw. athenischen Haushalt die attische Tragödie - anders als Homer - noch keine Referenzgröße war, auf die ein Redner oder ein öffentlicher Intellektueller wie Xenophon oder Isokrates Bezug zu nehmen hatte.

Wenn Euripides (und die Tragödie) bei Rednern - dies tun Lykurg, Aischines und Demosthenes - aufgerufen wird, hat dies bestimmte Voraussetzungen und - natürlich - Funktionen.

\subsection{Euripides in der Rhetorik des 4. Jh. v. Chr.}

Lykurg, um mit ihm zu beginnen, zitiert in der Rede gegen Leokrates eine bemerkenswert lange Partie (53 Verse!) aus Euripides' Erechtheus (fr. 360 Kannicht), die er mit deren Wert als Beispiel für eine patriotische Gesinnung rechtfertigt:

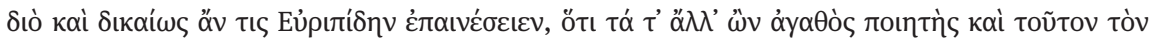

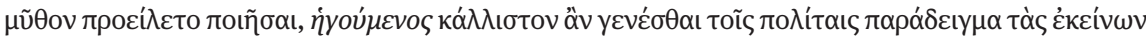

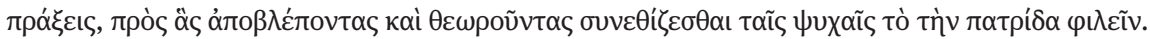
$[\ldots]$

15 Zur nur geringen Verwendung von Poesie in der Beredsamkeit des 4. Jh. siehe Perlman 1964, Wankel 1976, $1166 \mathrm{f}$.

16 Im Panathenaikos (168f.) findet sich lediglich ein pauschaler Hinweis auf die Tragödiendichter, die den Theben-Mythos behandelt haben. 
Daher könnte jemand auch wohl zu Recht Euripides loben, weil er, abgesehen davon, dass er auch im Übrigen ein guter Dichter ist [Parenthese: dieser Zusatz ist insofern erhellend, als er zeigt, dass eine solche Einschätzung des Euripides nicht selbstverständlich war], diesen Stoff für eine Tragödie wählte. Denn er war der Auffassung, dass ihre Taten für die Bürger das schönste Beispiel bieten. Wenn man auf jene blicke und sie aufmerksam betrachte, dann gewöhne man das Herz daran, das Vaterland zu lieben. [...] (Lyc. In Leocr. 100) $)^{17}$

Aus dieser Passage spricht eine Funktionsbestimmung der Tragödie, die deutlich eine politische Belehrung annimmt. Es ist dabei bedeutsam, dass Lykurg eine solche Annahme nicht etwa selbst vertritt, sondern Euripides zuschreibt (siehe meine Hervorhebungen). Er macht damit aus Euripides einen Dichter, der Tragödien schreibt, weil er darin die Möglichkeit sieht, seine Mitbürger durch das in den Dramen vermittelte Beispiel zu erziehen. Lykurg macht damit einerseits - natürlich - Euripides selbst zu einer Art von Patriot (weil er solche Verse gedichtet hat, die zu Patriotismus erziehen hier liegt die traditionelle antike und zumal für die Euripides-Exegese verwendete Annahme zugrunde, dass Dichter und Dichtung identisch sind ${ }^{18}$ ). Andererseits scheint er darin, Euripides eine politische Belehrung als Zielsetzung seiner Stücke zuzuweisen, die Linie einer politischen Deutung der attischen Tragödie fortzusetzen, die pointiert in Aristophanes' Fröschen entwickelt wird. Denn dort gibt auf die Frage des Aischylos, wofür man einen Dichter bewundern solle (1008), gerade Euripides die Antwort:

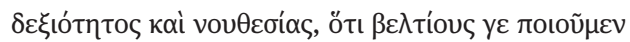

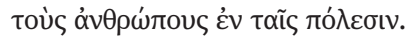

Um ihrer Klugheit und ihrer Belehrung willen, weil wir besser machen

die Menschen in den Städten. (Ar. Ra. 1009f.)

Dieses fiktive Selbstzeugnis aus einer Komödie lässt sich (natürlich ist fraglich, ob Lykurg gerade diese Operation unternahm) mit dem End- und Höhepunkt der von Lykurg zitierten Partie aus dem Erechtheus verbinden. Denn diese Rede der attischen Königin Praxithea, in der sie vor ihrem Gemahl Erechtheus ihre Tochter der Stadt zum Opfer gibt, endet mit einer pathetischen Apostrophe an Athen, die ihre opferbereite Vaterlandsliebe betont:

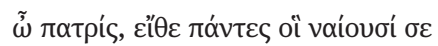

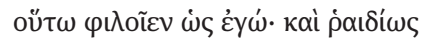

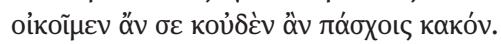

O Heimat, wenn doch alle, die dich bewohnen

dich so liebten wie ich; dann dürften leicht

wir dich bewohnen, und du littest kein Unheil. (fr. 360,53-55 Kannicht)

17 Text und Übersetzung nach Engels 2008.

18 Siehe hierzu Hose 1998. 
Da Lykurg auch Homer oder Tyrtaios mit ähnlicher politisch-didaktischer Zielsetzung zitiert (also auch diese mehr oder minder explizit als Erzieher einstuft), kann man - im Gefolge von Friedrich Blass - sein Interesse an Euripides und der Dichtung überhaupt in deren ,moralisch-didaktischem Gehalt' sehen. ${ }^{19}$ Wenn dies Lykurgs Blickwinkel auf die Tragödie konstituiert, ist es durchaus passend, dass es ihm darum gehen musste, deren Texte so authentisch wie möglich zu bewahren. Doch hierzu später.

Ein anderer Euripides tritt uns in den Reden des Aischines entgegen. Bunter und mannigfacher verwendet Aischines die Dichter und insbesondere Euripides in seiner Rede gegen Timarchos. Dort figuriert der Tragiker als allgemeiner Weisheitslehrer, er

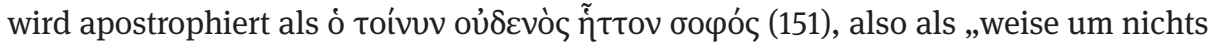
weniger als irgendeiner der Dichter“, d. h. einer der weisesten Dichter. Aischines be-

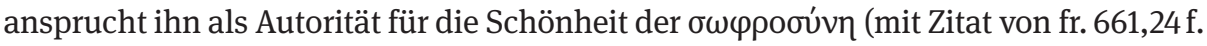
Kannicht ${ }^{20}$ aus der Stheneboia), zitiert eine längere Partie aus dem Phoinix (fr. 812 Kannicht), in der jemand den Titelhelden des Stückes gegen falsche Vorwürfe in allgemeiner Form verteidigt. Hierin will Aischines eine Analogie zu seinem eigenen Fall sehen. Und schließlich umschreibt er die Bedeutung der $\varphi \eta \mu_{\eta}$ (oder der Kunde) nicht nur mit einer Homer-Partie, sondern auch mit einem Euripides-Fragment aus einem nicht mehr kenntlichen Stück (128: fr. 865 Kannicht). Euripides ist also hier ein weiser Lebensbegleiter und Ratgeber, den der Redner soweit internalisiert zu haben scheint, dass ihm sogar - wohl ohne Absicht - Anklänge an Euripides-Verse in seine Reden einfließen, wie Eduard Fraenkel gezeigt hat. ${ }^{21}$

Auf den Euripides bei Aischines bezieht sich - polemisch - Demosthenes (andere Verweise auf den Tragiker finden sich im Corpus seiner Reden nicht). Aischines hielt seine Rede gegen Timarchos, der mit Demosthenes in der Gegnerschaft gegen die Makedonen verbunden war, 345. Zwei Jahre später klagt ihn Demosthenes wegen Verletzung der Pflichten in einer gemeinsamen Gesandtschaft zu Philipp an. ${ }^{22}$ Demosthenes nutzt seine Anklagerede (or. 19), um gerade die Dichter-Benutzung, die Aischines in der Timarchos-Rede betrieben hatte, gegen ihn zu wenden: ${ }^{23}$ Ohne Euripides zu zitieren, konstatiert Demosthenes, dass die von Aischines apostrophierte $\varphi \eta ́ \mu \eta$ von dessen Bestechlichkeit künde (244), und aus dem langen Zitat aus dem Phoinix zitiert Demosthenes maliziös nur den Schluss, den er wiederum gegen Aischines gerichtet sieht:

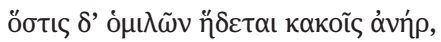

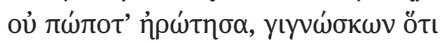

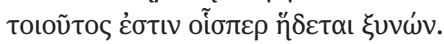

19 Blass 1898, 99.

20 Gerade diese Verse athetieren Diggle und Holford-Strevens, siehe Diggle 1998, 131.

21 Fraenkel 1960 mit Bezug auf Aischines 3, 144.

22 Zum Zusammenhang und der Datierung Paulsen 1999, 47-50.

23 Zum Einzelnen siehe Paulsen 1999, $241 \mathrm{f}$. 
Wenn jemand gern in schlechter Gesellschaft verkehrt, dann habe ich ihn gar nicht erst befragt, weil ich weiß, dass er ebenso ist wie diejenigen, mit denen er so gern umgeht. (Dem. or. 19, 245)

Sodann verspottet Demosthenes noch Aischines' (bekannte) Karriere als Tritagonist auf der Theaterbühne. ${ }^{24}$ Denn die berühmten Schauspieler Theodoros ${ }^{25}$ und Aristodemos, für die Aischines den dritten Part gegeben habe, hätten den Phoinix nie ge-

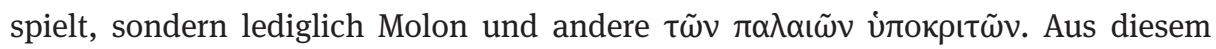
Spott werden zwei Dinge deutlich: Demosthenes verbindet Aischines' Euripides-Zitat mit dessen Schauspielerstatus, d.h. es ist nicht etwa Ausdruck von - lektüregetragener - Bildung, sondern Theaterpraxis. Und ferner wird mindestens dem Stück Phoinix, vielleicht aber sogar dem ganzen Euripides der Ruch gegeben, altmodisch zu sein, d.h. von ,alten' Schauspielern, die auf alte Stücke spezialisiert sind, aufgeführt zu werden.

Und noch in einer weiteren Partie (337) verbindet Demosthenes möglicherweise Euripides und die Schauspielkunst des Aischines: Um seine Zuhörer vor der Magie der offenbar eindrucksvollen Stimme des Aischines zu warnen, erinnert er an ein Debakel,

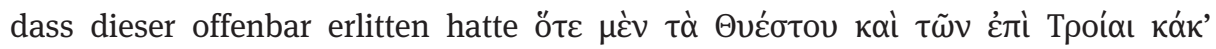

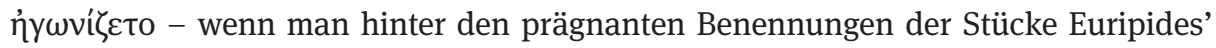
Kreterinnen und Troerinnen erkennt, ${ }^{26}$ läge hierin wiederum ein Zeugnis für die Aufführung von zwei Euripides-Dramen in einem Agon, freilich mit bösem Ausgang für Aischines, der, so Demosthenes, durch das Zischen und andere Unmutsäußerungen über sein Spiel aus dem Theater „verbannt“ ( $\dot{\varepsilon} \xi \varepsilon \beta \alpha \dot{\alpha} \lambda \lambda \varepsilon \tau^{\prime}$ ) wurde - also wohl zugleich seine Tritagonisten-Laufbahn aufgab - und fast gesteinigt worden wäre.

In der Kranzrede (or. 18), dem anderen Demosthenes-Text, der auf Euripides Bezug nimmt, sind diese Bezugnahmen wiederum an Aischines und sein Tritagonistentum geknüpft. Demosthenes kontrastiert mehrfach seine politische ,Rolle' mit Aischines' Theaterrollen. So sieht Demosthenes einen deutlichen Gegensatz zwischen seinem politischen Handeln und dem Auftreten des Aischines in nicht unbedeutenden Rollen als Kresphontes, Kreon oder Oinomaos (180), Rollen, die er (nicht unbedingt als Tritagonist) ${ }^{27}$ in Euripides' Kresphontes, Sophokles' Antigone und Oinomaos gegeben hatte. Bei der Aufführung des sophokleischen Oinomaos, hierauf braucht Demosthenes nur anzuspielen, war Aischines offenbar spektakulär als Oinomaos bei der Verfolgung des Pelops gestürzt und hatte sich damit nachhaltig lächerlich gemacht. ${ }^{28}$

Auch die zweite Partie, in der Demosthenes Euripides heranzieht, dient zur Kontrastierung seiner Leistungen bei Liturgien mit denen des Aischines, der statt öffentlicher Zeugnisse nur Euripides-Verse vortragen könne (267). Hierbei legt ihm

24 Siehe hierzu Schäfer 1885, 238-252.

$25 \mathrm{Zu}$ ihm Burkert 1975, 67-69.

26 So Schäfer 1885, 243f., Wankel 1976, 891; skeptisch Paulsen 1999, 297.

27 Siehe Wankel 1976, 891f.

28 Siehe etwa Vita Aischines 1,7 (ed. Dilts 1992), ferner Schäfer 1885, 248. 
Demosthenes (neben einem nicht identifizierbaren Fragment [TrGF 2, adesp. fr. 122 Kannicht-Snell] und einem Menander-Halbvers, Dysc. 926f.) den Eröffnungsvers aus

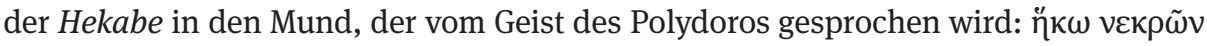

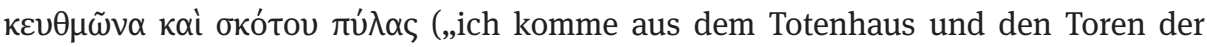
Finsternis“).

Betrachtet man nun das, was die Euripides-Rezeption bei Lykurg, Aischines und Demosthenes verbindet, so liegt es in der Bühnendimension dessen, was rezipiert wird. Es ist bezeichnend, dass Lykurg die Lehre, die man aus Euripides ziehen kann, als Lehre nicht einer Lektüre, sondern des Sehens, des Zuschauens benennt: $\pi \alpha \rho \alpha$ -

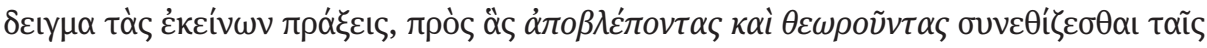

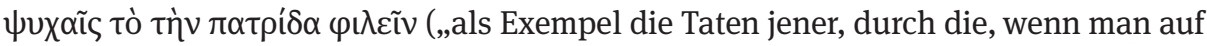
sie hinblickt und sie betrachtet, den Seelen die Vaterlandsliebe eingewöhnt werde“, Lycurg. 100,4-6). Für Aischines ist Euripides Gegenstand der Bühnenerfahrung, und bei Demosthenes figuriert Euripides als Opfer der Tritagonistenkunst seines Widersachers. Die Dimension, dass man Euripides lesen und sich über die Leseerfahrung aneignen könnte, also das Erlebnis, das der aristophanische Dionysos gemacht hat, ist offenbar den Rednern fremd.

Dies bedeutet, nimmt man diesen Befund ernst, dass Euripides über weite Strecken des 4. Jh. v. Chr. nur (oder wenigstens zum größten Teil) durch die Bühne und durch die Schauspieler vermittelt wurde. Als Text war damit das Corpus Euripideum nicht präsent und auch nicht Teil von entsprechenden literarischen Diskursen.

\subsection{Euripides im Theater des 4. Jh. v. Chr.}

\subsubsection{Wiederaufführungen und die Rolle der Schauspieler}

Welche Zeugnisse haben wir nun für diese Theaterexistenz des Euripides? Da sind zum einen die Nachrichten über die Aufführungen seiner Stücke, beginnend mit der postum durch den Sohn auf die Bühne gebrachten letzten Tetralogie. Ferner ist inschriftlich bezeugt durch die sog. Didaskalien-Inschrift (IG II ${ }^{2} 2320$ ) für die Jahre 341339, dass mithin an drei aufeinanderfolgenden Jahren (dies ist just der Ausschnitt, den das Inschriften-Fragment bietet) an den Dionysien als „altes Drama“ ein Stück des Euripides aufgeführt wurde. 341 war es eine Iphigenie, 340 der Orestes; für 339 ist der Titel weggebrochen. Mit diesen Aufführungen ist jeweils der Name des Schauspielers verzeichnet: 341 und 340 (339 ist der Schauspielername nicht erhalten) war es der auch sonst bekannte Neoptolemos von Skyros, ${ }^{29}$ der u.a. auf den denkwürdigen Hochzeitsfeierlichkeiten Philipps II. auftrat und die Ermordung des Makedonenkönigs miterlebte. ${ }^{30}$

29 Siehe Millis u. Olson 2012, 66.

30 Seinen Bericht hierüber hat Stob. 4,34,70 bewahrt. 
Die Dionysien 341 wie 340 standen durchaus im Zeichen dieses Mimen. Spielte er doch nicht nur den Protagonisten der beiden euripideischen Tragödien (und dürfte damit die Instanz gewesen sein, die für deren Wiederaufführung verantwortlich zeichnete); vielmehr trat er in dieser Funktion auch in je einem Stück der drei tragischen Trilogien ${ }^{31}$ auf, spielte also die Hauptrolle in vier Dramen vier verschiedener Dichter - und gewann 341 den Schauspielerwettbewerb. Seine Mitbewerber Thettalos und Athenodoros hatten dabei einen merkwürdigen strukturellen Nachteil. Da nur eine alte Tragödie aufgeführt wurde, konnten sie ihre Künste nur in je drei neuen Dramen zeigen. 340 half dieser Vorteil Neoptolemos nicht: Thettalos gewann den Agon.

Die Didaskalieninschrift eröffnet zudem eine interessante Perspektive auf die Euripides-Reprisen. Neoptolemos führte den Orestes 340 auf - im vorangegangenen Jahr hatte der Isokrates-Sohn Aphareus (TrGF 1,73, T 2 bzw. T 5) eine Trilogie mit den Stücken Peliaden, Orestes und Auge auf die Bühne gebracht, im Prinzip also (wir kennen nur die Titel der Stücke) Stoffe aufgegriffen, die Euripides bereits bearbeitet hatte. Er war damit, so kann man folgern, in eine Auseinandersetzung mit dem toten Vorgänger getreten, die auch in anderen Fällen für Tragiker des 4. Jh. v.Chr. bezeugt ist. Die damit implizit intendierte Überbietung war allerdings fehlgeschlagen, Aphareus wurde dritter und letzter im Agon; dass dies eine bittere Niederlage war, mag der Umstand andeuten, dass dies seine letzte Beteiligung am Tragiker-Wettbewerb war (TrGF 1,73, T 2). Wenn 340 nun das euripideische Original auf die Bühne kam, kann dies als Versuch gedeutet werden, dessen Stärken dem Publikum vor der Folie der misslungenen Neubearbeitung des Vorjahres zu demonstrieren. D. h. - mit Blick auf das eingangs zitierte Lemma aus dem 5. Bekkerschen Lexikon - Neoptolemos nutzte das prinzipiell größere Interesse des $\mathrm{Pu}$ blikums an neuen Tragödien, um dem Fehlschlag eines neuen Stücks ein Korrektiv, das $\pi \alpha \lambda \alpha ı$ òv $\delta \rho \tilde{\alpha} \mu \alpha$, gegenüberzustellen. Wir können also - zusammengefasst - im 4. Jh. v.Chr. einen dreifachen Wettbewerb konstatieren: Natürlich konkurrieren die neuen Dichter untereinander (1), doch sie suchen durch die Neubearbeitung von, alten' Stoffen den Wettbewerb mit den alten Dichtern (2), an den ein neuer Wettbewerb der alten Tragödien mit ihren Neubearbeitungen anschließen kann (3). ${ }^{32}$

An den Dionysien 340 geschah nun offenbar etwas Merkwürdiges. Denn Neoptolemos reüssierte trotz des ,Schlachtrosses“ Euripides’ Orestes nicht. Mochte auch das Euripides-Stück geeignet sein, Aphareus’ Versuch des Vorjahres zu überbieten, so gewann Thettalos den Schauspieler-Agon. Was war geschehen? Thettalos hatte den Protagonisten im ersten Stück der siegreichen Trilogie neuer Dramen gegeben, die Astydamas (der Jüngere) gedichtet hatte. Dieses erste Stück, der Parthenopaios, hatte

\footnotetext{
31 Die Struktur des Wettbewerbs war 341 und 340 gegenüber dem, klassischen` Ablauf geändert: Er begann mit einem Satyrspiel (das offenbar außer Konkurrenz stand), gefolgt von der alten Tragödie, hieran schlossen sich die drei Trilogien an.

32 Mit dieser Beobachtung gehe ich über die Rekonstruktionen (etwa von Seeck 1979a, 185f.) hinaus, die das Verhältnis von alter zu neuer Tragödie als lediglich ,einfachen' Agon ansetzen, in dem sich die neuen Stücke an den alten (die als vorgängig betrachtet werden) zu messen hatten.
} 
offenbar so viel Furore gemacht, dass die Athener dem Astydamas erlaubten, sich selbst eine Statue im Theater zu errichten. Dies tat der Dichter und verfasste dazu noch folgendes Epigramm:

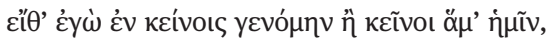

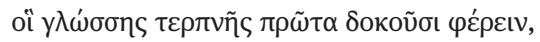

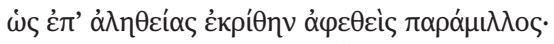

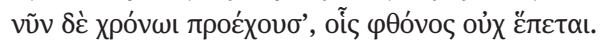

Wenn ich doch mit jenen geboren wäre oder jene zugleich mit mir, die den Ruhm haben, den ersten Preis der erfreuenden Rede davonzutragen, damit ich der Wahrheit entsprechend als gleichzeitig gestarteter Rivale beurteilt würde! Nun aber sind mir an Zeit sie voraus, denen kein Neid mehr folgt. (TrGF 1,60, T 2a) $)^{33}$

Dass aus diesem Epigramm ein Selbstwertgefühl des Astydamas spricht, es durchaus mit den alten Meistern aufnehmen zu können, diese aber - leider - einem direkten Wettbewerb entzogen seien, ist bereits der Antike deutlich gewesen. Man hat aus

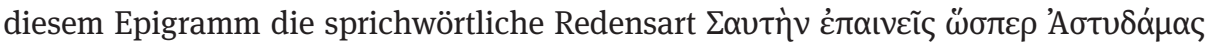

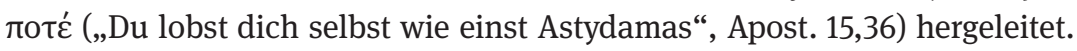

Gleichwohl: die Konstellation der Dionysien von 340 ist aufschlussreich. Zeigt sie doch, dass die Wiederaufführung alter Tragödien kein ,Selbstläufer‘ war, dass die alten Stücke, wenn sie dem sie initiierenden Schauspieler zum Erfolg im SchauspielerAgon verhelfen sollten, sich auch gegen gute neue Tragödien durchsetzen mussten.

Warum interessierten sich Schauspieler überhaupt so stark für alte Tragödien? Einen Hinweis gibt eine Bemerkung des Aristoteles in der Rhetorik: Im Zusammenhang seiner Erörterung der jeweils für eine (Rede-)Gattung geeigneten Form $(3,12)$ unterscheidet er zwei Arten des Vortrags: die schriftliche Darstellung (die er für be-

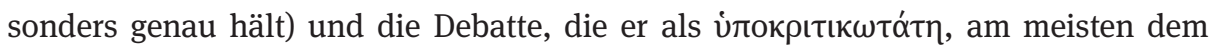

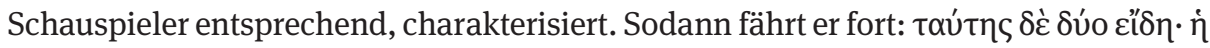

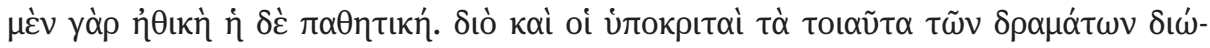
коvбı [...] („Von dieser aber gibt es zwei Arten: die charaktervolle und die emotionale. Deswegen suchen auch die Schauspieler nach dieser Art von Stücken [...]“, 1413b10f.). ${ }^{34}$

Mit dieser kurzen Bemerkung wird zweierlei deutlich: Aristoteles diagnostiziert ein besonderes Interesse der Schauspieler an Stücken, die ihnen Rollen mit spezifischen Darstellungsmöglichkeiten bieten, die sich mit den Begriffen „Charakter“ und „Pathos“ verbinden. Ferner ,warten“ die Schauspieler nicht einfach auf entsprechende Angebote, die ihnen durch neue Dichter gemacht werden könnten, sondern suchen

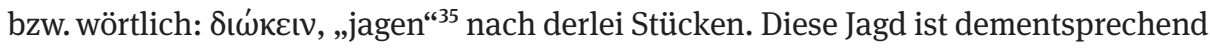
an die Initiative der Schauspieler geknüpft und weist auf deren Rolle bei der Wie-

33 Aus Pausanias Atticista, $\sigma 6$ Erbse, Übersetzung nach Gauly u. a. 1991, 135.

34 Griechischer Text nach Kassel 1976, Übersetzung nach Rapp 2002.

35 Siehe LSJ s.v. I. 1. 
deraufführung der ,alten“ Tragödien in den „Fasten“. ${ }^{36}$ Mit der Institutionalisierung der Wiederaufführung wäre demnach ein Ort geschaffen worden, an dem die ,Jagd“ nach geeigneten Tragödien ihr Ergebnis präsentiert.

Zudem, auch hierauf gibt Aristoteles in diesem Kapitel der Rhetorik einen Hinweis, entwickelte sich die Vortragskunst der Schauspieler im 4. Jh. v.Chr. dahingehend weiter, dass sie auch in bekannten Partien neue Akzente und Nuancen durch Ausdruckswechsel in der Sprache und in der Gestik erzielen konnten (Rhet. 3,12, 1413b21-29). ${ }^{37}$ Aristoteles, so hat Walter Burkert vermutet, konnte diese Beobachtungen in Theaterbesuchen selbst gewinnen, die er wohl um 350 machte. ${ }^{38}$

,Alte Tragödien, so kann man diese Bemerkungen zusammenfassen, waren aufgrund der in ihnen gegebenen Möglichkeiten eindrucksvoller Rollen per se für Schauspieler interessant, die dann durch ihre Darstellungskunst dem Altvertrauten neue Seiten abgewinnen konnten und damit auch das Grundinteresse des athenischen Publikums an Neuem zu bedienen wussten.

Hier ist sodann der Punkt, an dem die sog. Schauspieler-Interpolationen ihren Ausgang nehmen. Sie stellen sich als Instrument dar, den alten Stücken durch mehr oder weniger geschickte Zusätze neues Leben einzuhauchen.

\subsubsection{Schauspieler-Interpolationen}

Die Forschung hat diese Textveränderungen ja traditionellerweise geächtet, als Verfälschung des originalen Dichter-Wortes betrachtet und die berühmte Verordnung des Lykurg, die die Schauspieler auf das sog. Staatsexemplar verpflichten sollte, als segensreiche Intervention zugunsten der Originale bewertet. ${ }^{39}$ Dies hat freilich dazu

36 Vgl. oben S. 15.

37 Siehe hierzu Xanthakis-Karamanos 1980, 14 Anm. 3.

38 Burkert 1975.

39 Vgl. auch unten S. 29f. Als besonders gewichtig betrachtet Erbse 1992 diese Maßnahme für die Wiederherstellung eines ,reinen` Textes, die er jedoch an problematische Voraussetzungen knüpft: „Hat er (sc. Lykurg), um eine fortschreitende Verschlechterung aufzuhalten, lediglich den zu seiner Zeit erreichten Zustand des Textes festschreiben lassen? Das ist eine unbefriedigende Vermutung. Lykurgs Mühe hätte sich kaum gelohnt. In Wahrheit muß es Exemplare des Tragikertextes gegeben haben, die von Interpolationen frei waren [Hervorhebungen von M.H.]. Lykurg, Angehöriger des höchsten Adels und erster Staatsbeamter, hatte eher als jeder andere Bürger Athens die Möglichkeit, solche guten Texte zu erhalten. Vermutlich waren Abschriften hoher Qualität viel verbreiteter, als wir anzunehmen gewohnt sind. Andernfalls hätten ja die eigenwilligen Eingriffe der interpolierenden Schauspieler gar nicht aufgedeckt werden können [...]“ (Erbse 1992, 35). - Die Hervorhebungen machen die Postulate, die durch keine Belege gedeckt sind, deutlich, aus denen die conclusio abgeleitet wird: die vollständige Aufdeckung der Interpolationen durch diese Regelung Lykurgs, die sodann zu einem interpolationsfreien Staatsexemplar und einer entsprechend interpolationsfreien alexandrinischen Tragikerausgabe führen soll. 
geführt, dass eine systematische Arbeit an diesen Interpolationen in der griechischen Literatur ${ }^{40}$ mit der Ausnahme des Buchs von Page von 1934 unterblieben ist.

Diese Schauspieler-Interpolationen sind nun eine zweite große Auskunftsmöglichkeit über die Euripides-Wiederaufführungen im 4. Jh. v.Chr. Denn ihre Blütezeit sollte vor der lykurgischen Verordnung gelegen haben, d.h. wir können mit einer gewissen Plausibilität davon ausgehen, dass die in ,unserem', d.h. durch die alexandrinische Philologie stabilisierten und editorisch bearbeiteten Euripides-Text enthaltenen Interpolationen ,vor-lykurgisch“ sein sollten. Allerdings ist die Auswertung der Schauspieler-Interpolationen mit einem erheblichen methodischen Problem verbunden: Sie lassen sich nicht zweifelsfrei feststellen. Zu einem erheblichen Teil sind in den gegenwärtig führenden Euripides-Ausgaben entsprechende Diagnosen an Einschätzungen der jeweiligen Editoren geknüpft, was Euripides als Dichter zuzutrauen ist und was nicht. Zudem lehrt die Editionsgeschichte dieses Tragikers, dass selbst im Kreis derjenigen Herausgeber, die grundsätzlich umfänglichere Schauspieler-Interpolationen im Text annehmen, kein tiefgreifender Konsens über die entsprechenden Partien besteht. Dass der Euripides-Text überhaupt derartige Bearbeitungsspuren enthält, ist jedoch wenigstens unstrittig, wenn man die Euripides-Scholien als Testimonien hierfür würdigt. Denn in den Scholien wird wiederholt auf die Arbeit der Schauspieler hingewiesen. Eine aufschlussreiche Partie sei näher vorgestellt.

In Euripides' Orestes wird der berühmte Auftritt des Phrygers mit folgenden Chorversen eingeleitet:

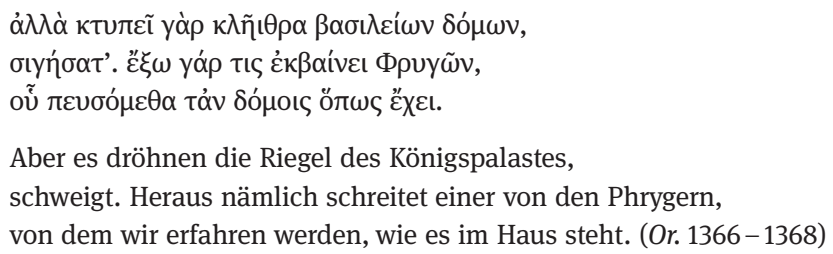

Hieran schließt sich der einen Botenbericht ersetzende Gesang (und Tanz) des phrygischen Sklaven an (1369-1502), der den Chor über Orests und Pylades' Attentat auf Helena unterrichtet. ${ }^{41} \mathrm{Zu}$ Beginn dieser Arie beschreibt der Phryger seinen Fluchtweg aus dem Haus:

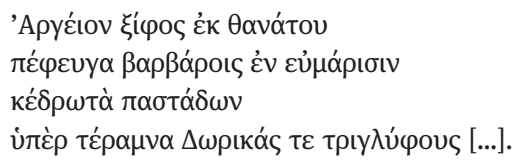

40 Anders steht es um die lateinische Literatur; hier sind in jüngerer Zeit grundsätzlichere Interpolationsforschungen $\mathrm{zu}$ insbesondere Plautus (Deufert 2002) und zur christlichen Literatur (Mülke 2008) vorgelegt worden, die jedoch teilweise auf erhebliche methodische Kritik stoßen (vgl. etwa Zetzel 2010).

41 Siehe hierzu Seidensticker 1982, 101-114. 
Vor dem argivischen Schwert entflohen bin ich dem Tod

in Barbaren-Mokkasins

über der Gemächer Zedern-Gebälk und dorische Triglyphe [...]. (Or. 1369-1373)

Daher hat die Forschung ${ }^{42}$ einerseits angenommen, der Phryger zwänge sich bei seinem Auftritt durch die (natürlich imaginären) Triglyphen des Palastes bei seinem Auftritt, d.h. er springe von einer Öffnung oberhalb der Türen des Bühnenhauses auf die Szene. Andererseits und in Stützung dieser Deutung, die zur Einstufung der Auftrittsankündigung als Schauspieler-Interpolation führt, ist das Scholion zu Or. 1366 genutzt worden:

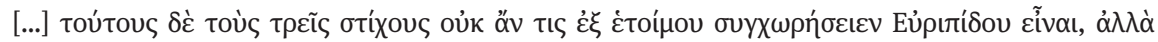

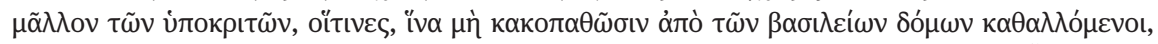

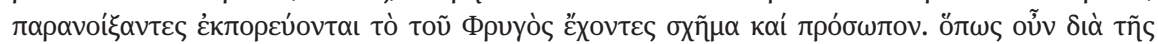

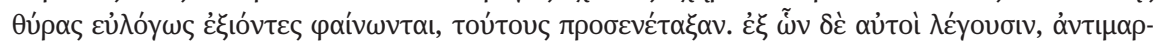

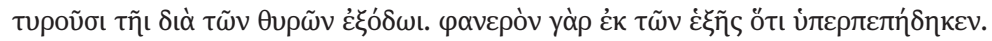

[...] Von diesen drei Versen dürfte man wohl nicht leichterdings zugeben, dass sie von Euripides stammen, sondern eher von den Schauspielern, die, damit sie sich nicht durch den Sprung vom Königspalast verletzen, stattdessen die Tür öffnen und in Haltung und Maske des Phrygers heraustreten. Damit sie nun offensichtlich plausibel durch die Tür heraustreten, haben sie diese Verse an dieser Stelle hinzugefügt. Aus dem, was sie selbst sagen, geben sie ein Gegenzeugnis zum Auftritt durch die Tür. Offensichtlich wird aus dem, was folgt, dass er (sc. der Phryger) darüber (sc. das Gesims) hinweggesprungen ist. (Schol. Eur. Or. 1366 Schwartz)

Dieses Scholion ist durchaus bedeutsam: Es zeigt das offenbar philologische Bemühen, mit einer diagnostizierten Diskrepanz im Euripides-Text umzugehen - der durch die Auftrittsankündigung benannten Auftrittsform für den Phryger durch die Bühnentür auf der einen Seite und der Betonung der Flucht durch die Triglyphe am oberen Bühnenhausteil auf der anderen Seite. Der Text des Scholions macht deutlich, dass die Lösung nicht etwa auf einer Evidenz oder historischen Nachricht über die Aufführungspraxis beruht, sondern auf einer philologischen Deduktion. ${ }^{43}$ Insofern ist in der Forschung nicht zu Unrecht umstritten, ob hier wirklich eine Schauspieler-Interpolation vorliegt oder nur eine philologische Vermutung die Athetese trägt. Von dieser Frage unabhängig ist jedoch der Wert des Scholions für die Existenz von SchauspielerInterpolationen schlechthin. Denn selbst wenn es sich hier um eine PhilologenKonstruktion handeln sollte, so beruht diese doch augenscheinlich auf der Kenntnis von Schauspieler-Eingriffen in den Text (denn sonst könnte eine solche Konstruktion nicht plausibel entstehen), ${ }^{44}$ die die Spielbarkeit eines Stückes gewährleisten oder erhöhen sollen.

42 Zur Forschungsgeschichte siehe Porter 1994, 192-199.

43 So auch - zu Recht - Erbse 1992, 39.

44 Anders Erbse 1992, 39, der hier lediglich den Unverstand des Interpreten diagnostiziert. 
Dass in den Scholien eine Vertrautheit mit den Praktiken der Schauspielkunst des 4. Jh. v. Chr. und des Hellenismus enthalten ist, zeigt das Scholion zu Orestes 57, das eine offenbar stumme Szene vor Einsetzen des Prologs bezeugt, die Schauspieler eingeführt haben:

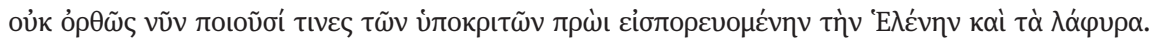

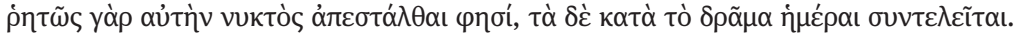

Nicht korrekt lassen jetzt einige Schauspieler in der Frühe Helena mitsamt der Beute in das Haus gebracht werden. Denn ausdrücklich sagt er (sc. Euripides), dass sie in der Nacht ausgeschickt (sc. vom Hafen in den Palast) worden ist. Die Geschehnisse im Drama werden an einem Tag vollzogen. (Schol. Eur. Or. 57 Schwartz)

Hiermit ist zwar kein Eingriff der Schauspieler in den Text verbunden, doch veränderten bzw. erweiterten sie das vom Text vorgezeichnete Bühnengeschehen. Wie auch immer man diese Erweiterung begründen mag, feststeht, dass sie eine ,Neuerung in ein vertrautes Stück einbrachte, die geeignet sein konnte, den ,alten' Orestes gegenüber den neuen Tragödien (wie dem Orestes des Aphareus von 341) ,konkurrenzfähig‘ in der Publikumsgunst zu halten.

In ähnlicher Weise ist es möglich, die immer wieder als Interpolation eingestufte Partie in der Medea 1056-1080 zu erklären (wenn man sie denn, was nicht zwingend zu erweisen ist, als Interpolation betrachtet): Hier würde dann der Monolog Medeas, in der sie ihren Kindsmord beschließt, um Zweifel und endlich Rückkehr zum Mordplan erweitert. Eine Notwendigkeit zu einer solchen Erweiterung ist erkennbar, da gerade Euripides' Medea im 4. Jh.v. Chr. als Ausgangspunkt für neue Bearbeitungen des Stoffs gedient hat: Man mag hier Neophron nennen, sicher gehört die Medea des Karkinos (TrGF 1,70, F 1e) in diese Reihe, ferner die Medea des Theodorides (TrGF 1, 78 A), die an den Lenäen 363 aufgeführt wurde.

Eine wichtige Zeugnisgruppe für den Reiz, der von Euripides’ Medea ausging, bilden Titel der Mittleren Komödie: Eine Medea ist bezeugt für Eubulos, Antiphanes und Strattis; ein Papyrus-Fragment (Pap. Lit. Lond. 77 = CGFP 350) bietet ein weiteres Medea-Drama, ${ }^{45}$ das möglicherweise eine Tragödien-Parodie oder sogar ein Satyrspiel war.

Es ist erkennbar, dass der Kindsmord mit seinen Implikationen der Angelpunkt der neuen Versionen war. So können wir aus einer Bemerkung in Aristoteles' Rhetorik (2,23, 1400b9 = TrGF 1,70, F 1e) erfahren, dass in Karkinos' Medea die Titelheldin sogar des Kindermordes angeklagt wurde, sie sich aber einer Verteidigungsstrategie bedient, die mit der Plausibilität operiert: ${ }^{46}$ Sie würde nicht ihre Kinder, sondern Jason getötet haben. Auf diesen Komplex verweist auch eine merkwürdige Notiz in einem durch einen Oxyrhynchos-Papyrus (5093, col. IV) überlieferten rhetorischen Text, die von einer Revision der Medea durch Euripides selbst weiß, mit der die ursprüngliche

45 Siehe Xanthakis-Karamanos 1980, 173-177.

46 Zu dieser ,neuartigen‘ Strategie siehe Solmsen 1931. 
Ökonomie des Stückes verändert worden sei. Denn die erste Fassung des Stückes habe

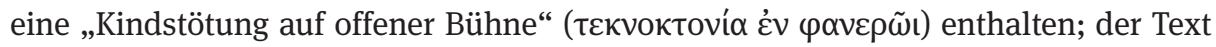
zitiert sogar zwei Verse eines Kindes, das von Medea mit dem Tod bedroht wird. Man kann hieraus schließen, dass zu einer bestimmten Zeit in der Antike zwei Versionen der euripideischen Medea im Umlauf waren, ${ }^{47}$ deren eine eben den Mord auf offener Bühne enthielt. ${ }^{48}$ Die bisherigen Interpretationen ${ }^{49}$ dieses Befundes sind von einer Doppelfassung ausgegangen, die Euripides selbst verfasste, also gleichsam eine recantatio seines früheren Stückes vorgelegt hätte (wie im Fall des Hippolytos, auf den der rhetorische Text des Papyrus selbst verweist). Genauso, wenn nicht aufgrund der Situation des 4. Jh. v.Chr. plausibler, könnte angenommen werden, dass eines der zahlreichen Medea-Stücke dieses aktiven Jahrhunderts, das besonders spektakulär den Kindermord thematisierte, unter den Namen des Euripides geriet (wie etwa der Rhesos), oder eine besonders weitreichende Schauspielerbearbeitung der euripideischen Medea vorgenommen wurde.

In einem solchen Umfeld ist die Verstärkung der Medea-Rolle durch eine intensivere Ausgestaltung des großen Entscheidungsmonologs im euripideischen Stück eine durchaus denkbare Maßnahme, die dieses Stück gleichsam konkurrenzfähig hielt. Einen ähnlichen Kontext kann man für die Phönissen rekonstruieren, ein gleichfalls unter dem Verdacht größerer Schauspieler-Interpolationen stehendes Drama. In der überlieferten Version stellen die Phönissen geradezu ein Kompendium des Labdakiden-Mythos dar: Die Belagerung Thebens durch die Sieben ist angereichert um eine noch lebende Iokaste und einen noch im Palast hausenden blinden Ödipus, am Ende zeichnet sich bereits der Konflikt um die Bestattung des toten Polyneikes zwischen Antigone und Kreon ab, und zudem geht Antigone mit Ödipus in die Verbannung.

Der Labdakiden-Stoff fand bei den ,neuen“ Tragikern reges Interesse. Die Didaskalien $^{50}$ bezeugen allein für die Dionysien 341 und 340 eine Antigone und den Parthenopaios des Astydamas sowie einen Ödipus des Timokles; hinzu kommen aus Karkinos’ Feder ein Amphiaraos und ein Ödipus, sowie ein Ödipus des Theodektas. Zu diesen und anderen ${ }^{51}$,neuen' Tragödien stellten sich die Antigone des Sophokles, deren Reprise mit Aischines als Kreon Demosthenes (or. 18, 267, vgl. or. 19, 246) be-

\footnotetext{
47 Siehe hierzu Luppe 2010 und Luppe 2011, der auf Pap. IFAO, PSP 248 hinweist, einen HypotheseisPapyrus, der von zwei Medeen weiß, und zudem auf Schol. Ar. Ach. 119 verweist, wo ein in der erhaltenen Medea nicht lokalisierbarer Vers aus der Medea zitiert wird (Eur. fr. 858 Kannicht), der aus der ,anderen' Medea stammen sollte. Merkwürdigerweise ist in der Debatte um die Papyrus-Nachricht bislang keinerlei Bezug genommen auf die älteren philologischen Debatten, die in der erhaltenen Medea die überarbeitete Version einer ersten Fassung erkennen wollen. Siehe hierzu die Übersicht bei Emonds 1941, $343 \mathrm{f}$.

48 Eine Rekonstruktion dieser Version bietet Mehl 2011.

49 Anders übrigens Colomo 2011 (die den Papyrus in den POxy ediert hat), die die Notiz für nicht geeignet hält, hieraus auf die Existenz eines Medea-Dramas mit Mord auf offener Bühne zu schließen. 50 Vgl. oben S. 20.

51 Vgl. aus den Adespota in TrGF 2: F 665 (Sieben oder Phönissen?), F 8, F 8a (Ödipus?).
} 
zeugt, $^{52}$ die beiden Ödipus-Dramen, in denen der Schauspieler Polos brillierte, ${ }^{53}$ wie auch offenbar die Sieben des Aischylos, deren Schluss von der Forschung mit großer Einhelligkeit als interpoliert betrachtet wird: Denn der dort (1005-1078) ausbrechende Streit zwischen Kreon und Antigone um die Bestattung des Polyneikes ist offenbar an der sophokleischen Antigone und den Phönissen orientiert und verfasst, ${ }^{54}$ um die Sieben ,konkurrenzfähig' mit diesen Stücken zu halten, d.h. wenigstens einen Ausblick auf den Stoff und den Konflikt zu geben, der im aischyleischen Stück schlicht nicht enthalten war. Man kann - pointiert - aus den Sieben schließen, dass es im 4. Jh. v.Chr. undenkbar oder wenigstens unüblich war, Tod und Bestattung der ÖdipusSöhne ohne den daraus bei Sophokles maßgeblich dargestellten Konflikt auf die Bühne zu bringen.

Innerhalb dieses Rahmens wird man grundsätzlich mit Anpassungen der alten Tragödien an diese Konkurrenz-Situation rechnen dürfen; ${ }^{55}$ allerdings ist - mit Ausnahme des Schlusses der Sieben des Aischylos - kein Konsens in Sicht, ob Partien wie Antigones berühmtes Kalkül ${ }^{56}$ in Sophokles' Stück oder bestimmte Partien der Phönissen entsprechende Interpolationen darstellen. In den Phönissen ${ }^{57}$ hat man nun insbesondere die Exodos als interpoliert betrachtet, da hier zwei Themen, die bei genauer Interpretation unvereinbar erscheinen, ausgespielt werden: Antigone will sowohl ihren blinden Vater Ödipus ins Exil begleiten als auch - gegen das Gebot des Kreon - ihren Bruder Polyneikes bestatten. Ersteres verbindet die Phönissen mit Sophokles' Ödipus auf Kolonos, Letzteres mit dessen Antigone. Es ist bereits von WolfHartmut Friedrich beobachtet worden, dass sich der Begräbnisstreit aus der Exodos herauslösen und die Vorweise, die auf ihn im vorausgehenden Stück gegeben werden, ohne weitere Verluste streichen lassen. ${ }^{58}$ Andererseits erscheint innerhalb der Antigone-Ödipus-Szene eine größere Partie (Phoen. 1737-1757) innerhalb ihres ergreifenden Klage-Duetts (1707-1757) als eine Art Dublette, ${ }^{59}$ so dass man nicht nur mit zwei verschiedenen Schlüssen, sondern auch Erweiterungen in diesen Schlüssen rechnen kann. Hiermit würden - hypothetisch - Euripides' Phönissen kombinierbar sowohl mit dem Ödipus auf Kolonos wie auch der Antigone - oder umgekehrt: sie ließen diese

52 Vgl. oben S. 19. Siehe auch Wagner 1995, 175.

53 Siehe Xanthakis-Karamanos 1980, 14 mit Anm. 2.

54 Siehe dazu Hutchinson 1985, 209-211.

55 Eine weitere Konstellation kommt im Fall der sophokleischen Dramen hinzu, wenn man den Überlegungen von C. W. Müller 1996/1999 folgt, der die bezeugte postume Aufführung des Ödipus auf Kolonos durch Sophokles' gleichnamigen Sohn als Teil einer Inhaltstrilogie annimmt, in der das Stück gemeinsam mit dem König Ödipus und der Antigone auf die Bühne kam. Für diese Inhaltstrilogie hätte Sophokles der Jüngere dann die beiden anderen Stücke zu der uns vorliegenden Form überarbeitet und teilweise erweitert (wie auch den $O C$ im 1. Epeisodion und in der Exodos).

56 Hierzu Rösler 1990.

57 Eine eher konservative Position nimmt - mit umsichtiger methodischer Reflexion - Mastronarde 1994, 39-49 ein. Eine Übersicht gibt Matthiessen 1994, 665-667.

58 Friedrich 1939, insbesondere 286.

59 Friedrich 1939, $292 \mathrm{f}$. 
Stücke als entbehrlich erscheinen, weil die Phönissen bereits ,alles` enthalten. Allerdings, dies ist trotz der eklatanten Widersprüchlichkeit der beiden Motivbereiche der Exodos zu konstatieren, bedeutet die Herauslösbarkeit des einen Bereichs nicht zwingend, dass dieser interpoliert ist, so dass, summa summarum, man das Vorhandensein von Schauspieler-Interpolationen im Euripides-Text zwar mit einiger Sicherheit konstatieren kann, deren konkreter Nachweis indes unsicher und an Prämissen über die Schreibweise des Euripides geknüpft ist, die nicht allgemein geteilt werden.

\subsubsection{Das sog. Staatsexemplar des Lykurg}

Auf die Arbeit der Schauspieler mit den Tragödientexten ,reagierte‘ Lykurg um 330, wenn man die entsprechende Notiz in Ps.-Plutarchs Leben der Zehn Redner für belastbar hält:

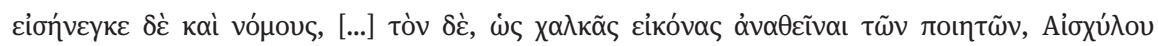

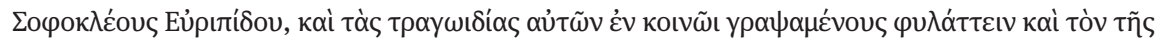

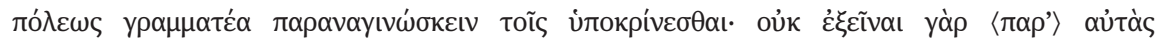

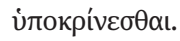

Er brachte auch Gesetze (sc. in die Volksversammlung) ein, [...] und das Gesetz, dass BronzeStandbilder der Dichter, nämlich des Aischylos, Sophokles und Euripides, aufgestellt werden und deren Tragödien aufgeschrieben in öffentlichem Besitz zu bewahren und dass der Stadtschreiber bei den Schauspielern (sc. den Text) parallel mitlese. Denn es sei nicht gestattet, gegen diese (sc. Tragödien) aufzuführen. (Ps.-Plut. Lycurg. 841F)

Dieses Gesetz zielt offenkundig auf eine Fixierung des Tragikerkanons: Die Aufstellung der Dichter-Standbilder macht diese zu ebenso festen Größen wie die Konstituierung eines ,öffentlichen‘ Textes, der den Kontrollmaßstab bei Aufführungen bilden soll, indem mit ihm der Text der Schauspieler abgeglichen werden muss. ${ }^{60}$ Man mag sich fragen, warum Lykurg die Sicherung der Authentizität der Tragiker-Texte so wichtig war. Ihr Gebrauch in der Rede gegen Leokrates ${ }^{61}$ gibt hierfür die Erklärung: Wenn das ,Tragiker-Wort` als moralische oder politische Richtlinie Gewicht haben sollte, war es von entscheidender Bedeutung, dass unzweifelhaft ein nicht-interpolierter Text, der das ganze Gewicht der alten Dichter verkörperte, auf die Bühne kam, nicht aber eine Hinzufügung von Schauspielern.

Welche Bedeutung die der Schauspieler-Kontrolle zugrunde liegende Textsammlung, das sog. athenische Staatsexemplar, für die Überlieferungsgeschichte der Tragiker hatte, ist angesichts fehlender belastbarer Nachrichten - abgesehen von der

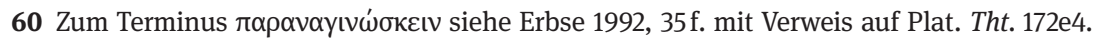
61 Vgl. oben S. $16 \mathrm{f}$. 
berühmten Ausleihe des Exemplars nach Alexandria ${ }^{62}$ - dunkel. Während etwa Hartmut Erbse in diesem Exemplar und seiner Benutzung die Garantie für einen im Wesentlichen interpolationsfreien Tragikertext sah, ${ }^{63}$ stufte Ulrich von WilamowitzMoellendorff seine Bedeutung gering ein: „Das athenische Staatsexemplar hat keine erkennbare Wirkung gehabt; wahrscheinlich überhaupt keine, und gewiss mit Recht."64

\subsubsection{Euripides-Rezeption bei Platon und Aristoteles}

Bis zu diesem Punkt haben wir die Euripides-Rezeption als Rezeption eines Bühnenwerks erfahren, d.h. vom ursprünglichen Sitz im Leben der Dramen her. Natürlich wird im Lauf des 4. Jh. v.Chr. durch die sich ständig erhöhende Bedeutung des Buchs die Bedeutung dieser Rezeptionsform abgeschwächt. Bei Platon, in dessen Dialogen Euripides gelegentlich zitiert wird, ${ }^{65}$ dominiert freilich noch das Bühnenerlebnis eines dramatischen Textes, auch wenn an wesentlich zahlreicheren Stellen durchscheint, dass Platon das euripideische Gesamtwerk gut kannte. ${ }^{66}$ Selbst in abstrakteren Partien wie in den Gesetzen wirkt die Bühne weiter:

Dort erläutert der Athener, dass die Aufgabe der Gesetzgeber, in der Polis das Recht durchzusetzen, durch die Sozialisation der Politen erleichtert wird, die durch die Dramen erfahren, was den Göttern ein Gräuel und was schändlich ist:

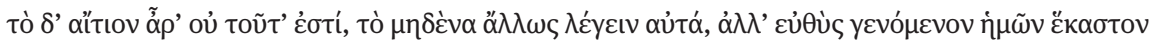

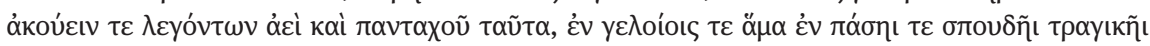

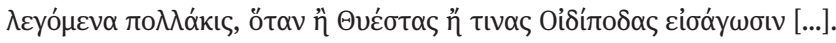

Ist aber diese Wirkung nicht darin begründet, dass niemand anders davon spricht, sondern dass jeder von uns gleich von seiner Geburt an stets und überall diese Ansicht hört, sowohl im Lustspiel als auch im tiefen Ernst der Tragödie, wo sie oft genug vorgetragen wird, wenn die Dichter einen Thyestes oder einen Ödipus auftreten lassen [...]. (Plat. Lg. 7, 838c) ${ }^{67}$

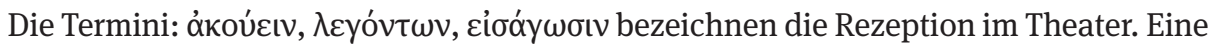
Wende in der Rezeptionshaltung nicht nur zu Euripides, sondern zum gesamten Drama bedeutet natürlich Aristoteles, der in der Poetik dezidiert die ,Bühnenhaftigkeit‘ trotz Anerkennung ihrer Wirkung auf den Zuschauer aus der Leistung der Poesie ausklammert:

62 Bezeugt bei Galen, In Hippocr. ep. III comm. 2,4 (= CMG 5, 10,2,1).

63 Zuletzt Erbse 1992, 34-36.

64 Wilamowitz 1880/1935, 25 Anm. 3.

65 So werden im Gorgias (484e, 485e, 486b) von Kallikles Verse aus der Antiope zitiert, im Symposion (177a) ein Vers aus der Melanippe, im Ion (533d) ein Vers aus dem Oineus.

66 Hierzu insgesamt Sansone 1996.

67 Text und Übersetzung nach Schöpsdau 1977. 


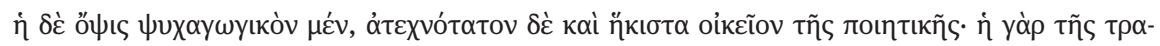

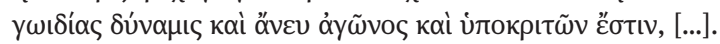

Die Inszenierung vermag zwar die Zuschauer zu ergreifen: sie ist jedoch das Kunstloseste und hat am wenigstens etwas mit der Dichtkunst zu tun. Denn die Wirkung der Tragödie kommt auch ohne Aufführung und Schauspieler zustande, [...]. (Arist. Poet. 6, 1450b16-19) ${ }^{68}$

Diese Trennung der Tragödie von ihrer Aufführung stellt, wie aus dem Vorangehenden deutlich wird, einen radikalen Bruch mit der kulturellen Praxis des 4. Jh. dar; von hier aus ist die Auseinandersetzung mit dem Drama und insbesondere mit Euripides als textuellem Phänomen explizit theoretisch gerechtfertigt.

Es ist zudem nachvollziehbar, dass Aristoteles eine Präferenz von Dramatikern, die stärker zur Lektüre als zur Aufführung geeignet erscheinen, in der Rhetorik $(3,12)$ entwickelt. ${ }^{69}$ Freilich, dies ist zu konstatieren, bedeutet das nicht etwa, dass es schon im 4. Jh. v.Chr. ,Lesedramen' gegeben hätte. ${ }^{70}$

\section{Euripides im 3. Jh. v. Chr.: Ehreninschrift für einen Schauspieler}

Natürlich tat die durch Aristoteles fundierte neue Betrachtung der Tragödie als Text deren Bedeutung für die Bühne zunächst keinen Abbruch. Allerdings ergibt sich, wenn man die Bühnenpräsenz nachzeichnen will, ein Problem. Am Ende des 19. Jh. fand man in Tegea eine Marmorbasis, Überrest offenbar einer Ehrenstatue eines Schauspielers (sein Name ist nicht erhalten) im Theater der Stadt, die eine interessante Auflistung seiner Erfolge inschriftlich festhielt:

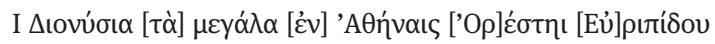

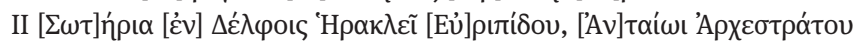

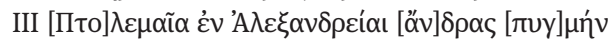

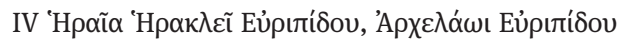

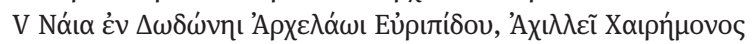

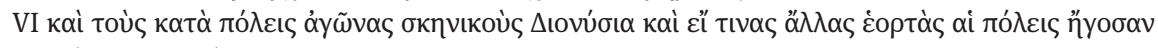

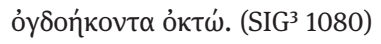

Die Inschrift ${ }^{71}$ teilt eine stolze Anzahl von Erfolgen an den namentlich genannten ,Kranz'-Spielen, also Agonen, bei denen der Sieger durch einen Kranz symbolisch belohnt wurde, und summarisch aufgeführten anderen Agonen mit, bei denen in der Regel ein Geldpreis die Belohnung für den Sieg bildete. Die genannten Spiele geben

68 Text nach Kassel 1965, Übersetzung nach Fuhrmann 1994.

69 Vgl. oben S. $22 \mathrm{f}$.

$70 \mathrm{Zu}$ Chairemon und den ,Anagnostikoi‘ siehe weiterhin Crusius 1902.

71 Siehe hierzu Herzog 1901. 
zudem Anhaltspunkte für die Zeit der Siege (und mittelbar für die Datierung der Inschrift). Die Zerstörung Dodonas durch die Aitoler 219 v.Chr. bildet einen Terminus ante quem, die Einrichtung der Soteria bzw. Ptolemaia 274 v. Chr. einen Terminus post quem. Die Siege gehören also in die Zeit zwischen ca. 280 und 220 v. Chr. Sie zeigen einen erfolgreichen Schauspieler, der auch im Faustkampf gewonnen hat. Dass dies zusammenpassen kann, zeigt die Liste der Stücke, mit denen er siegte: Orestes, Herakles, Archelaos, Achilleus, Antaios - offenbar Stücke, in denen in der Regel ein ,starker` Protagonist gebraucht wurde. Dies erfüllte der Geehrte, wusste aber auch als kranker Orestes zu überzeugen.

Da nun die Auswahl ,alter‘ Tragödien in einem Agon beim Schauspieler lag, hatte sich dieser Stücke ausgesucht, die ihm buchstäblich auf den Leib geschriebene Rollen boten. Es konnte also, so darf man schließen, nicht nur die große dramatische Szene, die etwa der Medea-Monolog bot, sondern auch die physische Anforderung ein Gesichtspunkt für die Auswahl von (Euripides-)Reprisen sein. Deutlich wird zudem aus der Inschrift, dass Euripides im 3. Jh. v.Chr. an allen großen szenischen Agonen der griechischen Welt gespielt wurde. ${ }^{72}$ Denn die Anzahl dieser Wettbewerbe war, soweit sich ermitteln lässt, seit dem späten 5. Jh. v.Chr. kontinuierlich angewachsen: Kann man um 400 v.Chr. von 11 Agonen, an denen sicher Dramen aufgeführt wurden, und zusätzlichen 28, an denen dies wahrscheinlich der Fall war, ausgehen, so lassen sich für die Zeit um 340 v.Chr. 23 Feste mit nachgewiesenen Dramenaufführungen und weitere 55 ansetzen, an denen mit einiger Wahrscheinlichkeit produziert wurde. ${ }^{73}$ Wie die Inschrift aus Tegea zeigt, standen auch außerhalb von Athen hierbei - vielleicht sogar in der Regel - alte Tragödien auf dem Programm. ${ }^{74}$

\section{Euripides im späten Hellenismus und in der Kaiserzeit}

Freilich wird diese Beleglage im späten Hellenismus und schließlich in der Kaiserzeit immer dürftiger. Explizit bezeugt sind Dramenaufführungen für die athenischen Panathenäen des 1. Jh. n. Chr. (neue Tragödie), die Sebasta in Neapel im Jahr 42 n. Chr. (neue Komödie), die Kaisarea auf dem Isthmos 127 n.Chr. (Komödie, wahrscheinlich auch Tragödie), die Museia von Thespiai im 2. Jh. n.Chr. (alte Tragödien und Komödien, neue Tragödien und Komödien) und den musisch-dramatischen Agon in Aphrodisias am Ende des 3. Jh. n. Chr. (alte Komödien und neue Tragödien und Ko-

$72 \mathrm{Zu}$ den Theatern im 4. Jh. v. Chr. siehe die Beiträge in Csapo et al. 2014. Eine erste Orientierung kann weiterhin Webster 1963 bieten.

73 Zahlen nach Csapo 2010, 103; vgl. Taplin 2012, 239.

74 Siehe Heldmann 2000, 190 mit Anm. 29. Vgl. dazu auch Tedeschi 2002. 
mödien).$^{75}$ Merkwürdigerweise fehlen Nachrichten über ein Fortleben des DramatikerAgons an den athenischen Dionysien der Kaiserzeit (der Dithyramben-Wettbewerb wird dagegen erwähnt). ${ }^{76}$

Dieser bemerkenswerte Befund benötigt freilich einige Korrektive. Denn mit dem Schwinden des Tragikeragons einher geht offensichtlich ein Sich-Verstärken anderer Rezeptionsformen von Tragödie und zumal der Dramen des Euripides. So wächst auf der einen Seite die Bedeutung von Buch und Lektüre. Erleichtert durch die Arbeit der hellenistischen Philologie, die Editionen wie Kommentare bereitstellt, ${ }^{77}$ wird der Euripides-Text fast ein Allgemeingut, das zum Rüstzeug der Bildung gehört. Prägnant kommt dies durch eine Bücherspende zum Ausdruck, die eine um 100 n.Chr. zu datierende Inschrift im Piräus verzeichnet: offenbar hatte ein Ephebenjahrgang seinem Gymnasium eine Euripides-Teil-(oder Gesamt-)Ausgabe dediziert. ${ }^{78}$ Prägnant zeigen dies auch die Papyrus-Funde aus Euripides-Stücken, die Texten des Schulunterrichts entstammen. ${ }^{79}$ Euripides ist damit als ,textuelles Phänomen' fest im Kulturbetrieb des Hellenismus und der Kaiserzeit verankert.

Auf der anderen Seite stehen Veränderungen im Theaterbetrieb, die mit der starken Expansion des Theaterbauens im Mittelmeerraum einhergingen. ${ }^{80}$ Diese Bautätigkeit führte dazu, dass ein monumentales Steintheater zum Standard-Inventar einer hellenistischen Polis ${ }^{81}$ und später einer Stadt der Kaiserzeit gehörte. Man kann hieraus rein rechnerisch auf einen ungeheuren Bedarf von im Theater Aufführbarem schließen, der mit einer auch in der Kaiserzeit stetig wachsenden Zahl von Festen mit Agonen auch musischen Charakters begleitet und quasi verschärft wurde. So könnte es allein vom 1. bis zum Ende des 3. Jh. nach einer Schätzung von Peter Herz 3-4000 derartige Spiele im Imperium Romanum gegeben haben, d. h. mehr als zehn Spiele pro Jahr mit entsprechenden musischen Agonen. ${ }^{82}$

Dass die zahlreichen Theater und Agone adäquat bestückt werden konnten, verdankt sich wohl mindestens teilweise verschiedenen Entwicklungen, die bereits im Frühhellenismus einsetzen:

75 Siehe die Einzelnachweise bei Heldmann 2000, 191f. Die Inschrift von Aphrodisias (Text und Übersetzung bei Roueché 1993, 166 -168) teilt auch die Summen mit, die der Wettbewerb den Tragöden einbrachte: dem Sieger 1500, dem Zweiten 600 und dem Dritten nur noch 250 Denarii.

76 Vgl. Mette 1977, 100; Heldmann 2000, 190.

77 Siehe hierzu Falkner 2002 mit weiterer Literatur, der zudem den Einfluss der Bühnenerfahrung auf die Arbeit der Philologen diskutiert.

78 IG II/III $2363=\operatorname{TrGF} 5$, T 7a (mit weiterer Literatur).

79 Siehe etwa POxy. 2337 (aus dem Orestes), PSI 13,1303 (aus den Phönissen); die Beispiele lassen sich leicht vermehren. Auch das Titelverzeichnis POxy. 2456 (= TrGF 5, T 8) dürfte, da auf der Rückseite eines Steuer-Registers geschrieben, in den Kontext des Unterrichts gehören.

80 Siehe hierzu insgesamt v. Hesberg 2009. Einen Gesamtüberblick zum Hellenismus gibt Brüggemann 2011.

81 Zu diesem Aspekt speziell Le Guen 1995.

82 Herz 1990, 177. 
1) Der Schauspieler-Betrieb professionalisierte sich; es entstanden zu Beginn des 3. Jh.v. Chr. ,Gilden', Zusammenschlüsse, an die sich Städte wenden konnten, wenn sie für ihre Agone histrionischen Bedarf hatten. Diese Entwicklung ging einher mit einer garantierten Reise- oder Bewegungsfreiheit der Schauspieler zunächst in Griechenland (dort um 279 v.Chr. durch ein Dekret der Amphiktyonie festgelegt), die sie risikolos in alle möglichen Städte brachte. ${ }^{83}$

2) Die ,konzertante“ Präsentation gewann an Bedeutung gegenüber der szenischen. Henner von Hesberg hat vor einiger Zeit darauf hingewiesen, dass in Athen bereits am Ende des 4. Jh. v.Chr., auf Veranlassung des Demetrios von Phaleron, Rhapsoden im Theater auftraten, dass in Alexandria anlässlich der Vergöttlichung Alexanders im Theater die Werke Homers und Hesiods rezitiert wurden. ${ }^{84}$ Das ,Monopol' des Dramas auf Theater-Aufführung war also gefallen, und wenn Hesiod oder Lyriker im Theater rezitiert wurden, war der Schritt, dass dies auch Tragiker tun konnten, nicht groß. In gewisser Hinsicht war die konzertante Aufführung der Tragödie oder von Teilen der Tragödie bereits im 5. Jh. v.Chr. in privaten Kontexten vorbereitet. So bittet in Aristophanes' Wolken der alte Strepsiades seinen Sohn um den Vortrag von Simonides-Liedern oder Aischylos - und muss von diesem eine Rhesis aus Euripides' Aiolos (1371) hören. In den Fröschen schließlich wird gar Euripides selbst als Deklamator seiner Texte vor „Kleiderdieben“, „Taschendieben“, „Vatermördern“ und „Einbrechern“ imaginiert, die von dieser Darbietung in manische Verzückung geraten:

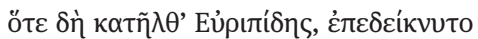

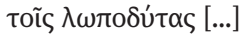

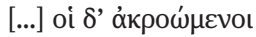

$[\ldots]$

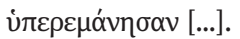

Als nun Euripides heruntergekommen war, gab er eine Probe seiner Kunst den Kleiderdieben [...]. Die hörten genau zu [...] und gerieten vor Begeisterung außer sich [...]. (Ar. Ra. 771f., 773, 775) ${ }^{85}$

Geradezu eine Spiegelung dieser Szene bietet Lukian ${ }^{86}$ in seiner Schrift Wie man Geschichte schreiben muss: ${ }^{87}$ Dieser Text ${ }^{88}$ wird mit einer Anekdote eingeleitet, die eine

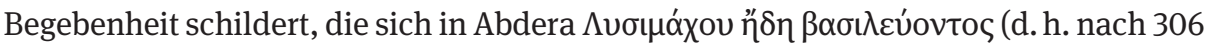
v.Chr.) zugetragen haben soll: Die Abderiten seien von einer pathologischen Euripides-Manie befallen worden, die der Tragödien-Schauspieler Archelaos verursacht

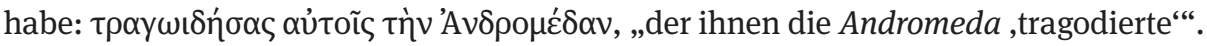

83 Siehe hierzu insgesamt Pickard-Cambridge 1968, 279-305; neuere Literatur bei v. Hesberg 2009, 293 mit Anm. 66.

84 v. Hesberg 2009, $291 \mathrm{f}$.

85 Vgl. hierzu auch Hunter 2009, 10 -12.

86 Siehe hierzu insgesamt Seeck 1990.

87 Siehe zu diesem Text insgesamt Free 2015.

88 Im Folgenden ist herangezogen Homeyer 1964, siehe außerdem den Beitrag von Manuel Baumbach in diesem Band, S. $184 \mathrm{f}$. 
Diese Formulierung macht augenscheinlich keinen Unterschied zwischen dem Stück und der (Haupt-)Rolle, und es ist daher auch nicht klar, ob prägnant die szenische Aufführung des gesamten Dramas mit zusätzlichem Personal oder die ,konzertante، Solo-Darbietung gemeint ist. Dass in jedem Fall an eine öffentliche Darbietung im Theater gedacht ist, machen die Begleitumstände deutlich. Denn wenn die Abderiten in toto von der Manie befallen werden, müssen sie auch in toto der Darbietung ausgesetzt worden sein; da Lukian zudem besonderen Wert auf die Zeitangabe der Ar-

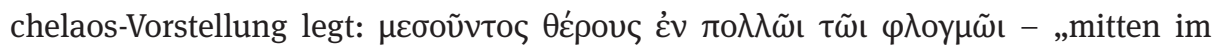
Sommer bei glühender Hitze“ -, ist damit angedeutet, dass diese Vorstellung in einer Zeit stattfand, in der man klüglich besser keine Theaterdarbietungen durchführt. D. h. die Präsentation von Euripides' Andromeda bei den Abderiten ist nicht Teil eines kultischen Festprogramms mit szenischen Spielen, sondern ein einmaliger Vorgang ohne den üblichen Festrahmen. ${ }^{89}$ Wie auch immer man die potentielle Historizität dieses Ereignisses betrachten mag, ist damit angezeigt, dass in der Zeit des 2. Jh. n.Chr. eine epideiktische Dramen-Präsentation ohne explizite Einbindung in einen festlich-kultischen Zusammenhang keine Besonderheit darstellt. Man darf also eine Autonomie des Theaters vom Kult in der Kaiserzeit und wohl auch im Hellenismus annehmen.

Eine weitere Tendenz tritt in der Kaiserzeit deutlicher hervor: die zunehmende Partikularisierung der ,Kunst‘ in den Spielen. War für das 4. Jh. v. Chr. ein Wettbewerb der Schauspieler kennzeichnend, in dem deren Fähigkeiten aus den Dramen im Agon zu extrapolieren war, so bildet sich - es ist nicht deutlich, ab wann - spätestens in der Kaiserzeit ein separates Wettkampfwesen für die Schauspielkunst in ihren Sparten aus. Deutlich wird dies etwa aus einer Ehreninschrift in Argos (wohl 2. Hälfte des 2. Jh. n.Chr.) für C. Iulius Bassus, die dessen Erfolge bei zahlreichen Spielen in den Kategorien „Herold“, „Tragöde“ und „Komöde“ nennt. ${ }^{90}$ Dies führt darauf, dass z. B. an den Panathenäen oder den Nemeen etc. der Kaiserzeit die Schauspielkunst in Sparten - analog zu den gymnischen Wettbewerben - im Agon dargeboten wurde, und es ist kaum anders zu denken, als dass hierbei nur Teile von Dramen zur Darbietung kommen konnten. Ob und inwiefern hier auch die Veränderung der Bühne seit dem

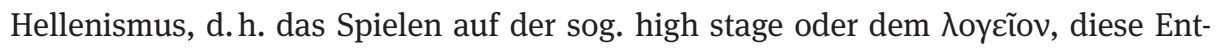
wicklung unterstützte, ist eine nicht sicher zu beantwortende Frage. Hingewiesen sei immerhin auf die These von Carl Robert, die den Ort der Schauspieler vom späten 4. Jh. v.Chr. an auf diesem herausgehobenen Platz des Theaters lokalisiert und - mit Blick auf die ,neuen` Tragödien dieser Zeit - eine Loslösung von der Orchestra annimmt. ${ }^{91}$

Unter solchen Rahmenbedingungen erscheinen die Nachrichten, die „Aufführungen“ von Euripides-Stücken in der Kaiserzeit melden, stärker Rezitationen von

$89 \mathrm{Zu}$ dieser bei Lukian geschilderten Anekdote siehe auch unten Manuel Baumbach in diesem Band. 90 Moretti 1953, 215f. (Nr. 74), dazu Heldmann 2000, 192.

91 Robert 1897, 447-453. 
Teilen von Stücken als komplette Inszenierungen anzuzeigen. Deutlich macht dies etwa Plutarch in der Crassus-Vita - eine Notiz, die Bruno Snell (TrGF 1, 20) in seiner Zusammenstellung der ,Didascaliae‘ ohne weitere Hinweise als für eine Aufführung der Bakchen in Parthien im Jahr 53 v.Chr. geltend macht. Plutarch schreibt jedoch:

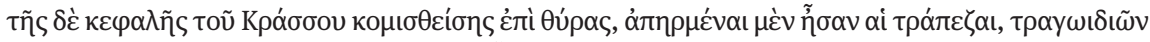

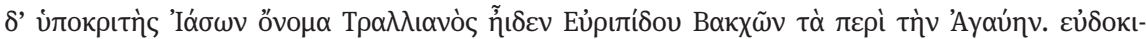

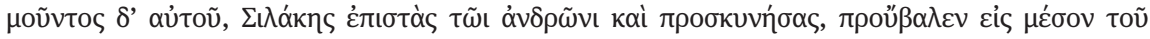

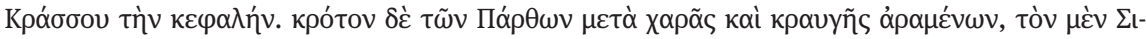

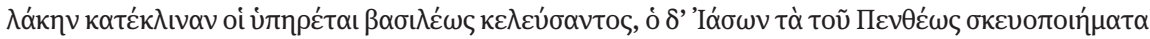

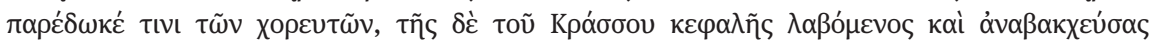

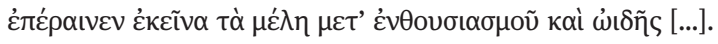

Als da der Kopf des Crassus an die Türen gebracht wurde, waren [sc. in der Residenz des PartherKönigs Orodes] die Tische gerade abgetragen, und ein tragischer Schauspieler, Jason von Tralleis mit Namen, sang aus den Bakchen des Euripides die Szene um Agaue. Während er mit Beifall überschüttet wurde, trat Silakes [einer der parthischen Feldherren, die Crassus besiegt hatten] in die Tür des Saales, erwies den Fußfall und warf den Kopf des Crassus mitten hinein. Während die Parther unter lautem Freudengeschrei Beifall klatschten, wiesen die Diener auf Befehl des Königs dem Silakes einen Platz an, Jason aber übergab die Maske des Pentheus einem der Chormitglieder, packte den Kopf des Crassus und sang in höchster bakchischer Begeisterung die Verse [...]. (Plut. Crass. 33,3f.) $)^{92}$

Es folgen die Verse 1169f. aus den Bakchen. Diese Begebenheit findet augenscheinlich im Speisesaal statt; nach dem Mahl - die Tische sind abgetragen - trägt ,konzertant (anders ist es aufgrund der Räumlichkeit nicht denkbar) der Schauspieler Jason im

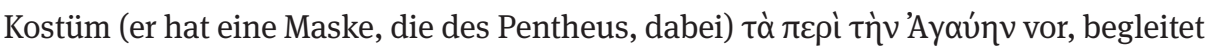
von einem Chor. Da es in den Bakchen keine Szene gibt, in der Pentheus und Agaue gleichzeitig zugegen sind (anders natürlich im Botenbericht), ist nicht klar, welche Partie Jason vorträgt. Wenn er sodann die Maske des Pentheus ablegt, ohne die der Agaue aufzusetzen, müsste dies bedeuten, dass Partien, in denen diese auftritt, gar nicht für die Präsentation vorgesehen waren. Jason ,improvisiert‘ also, er nutzt die Gelegenheit, den Kopf des Crassus als Requisit für die Agaue-Szene der Exodos einzusetzen und damit wirkungsvoll Darbietung und Gegenwart des Sieges über Crassus zu verbinden. Der Plutarch-Text spricht also nicht von einer Aufführung der Bakchen bei den Parthern, sondern der Darbietung einer Szene aus den Bakchen, und er legt nahe, dass Jason auch Szenen aus anderen Stücken vorzuführen gedachte, sein Programm aber aus ,aktuellem Anlass‘ änderte.

In entsprechender Weise sind Suetons Angaben über Neros histrionische Aktivitäten $\mathrm{zu}$ verstehen: tragoedias quoque cantavit [...] inter cetera cantavit Canacem parturientem, Orestam matricidam, Oedipodem excaecatum, Herculem insanum (,er trat auch in Tragödien auf [...], u.a. trat er in ,Die Niederkunft der Kanake‘, ,Der Muttermörder Orest‘, ,Der geblendete Ödipus‘ und ,Der rasende Hercules` als Sänger

92 Text nach Ziegler u. Gärtner 1994; Übersetzung nach Ziegler 1955. 
auf“, Suet. Nero 21,3). ${ }^{93}$ Die Zusätze zu den Namen machen deutlich, dass Nero nur die jeweiligen Glanzpartien, die die Zusätze anzeigen, bestritt. Auch die Nachrichten zu Neros agonistischen Aktivitäten auf seiner großen Griechenland-Reise weisen darauf, dass er an Tragöden-Agonen teilnahm und dort nur einzelne Partien aus Dramen vortrug. ${ }^{94}$

Diese hohe Bedeutung der ,besonderen' Szenen dramatischer Texte für Aufführungen zeitigte offenbar zwei Nebeneffekte. Der eine lag darin begründet, dass bei den lyrischen Partien in vielen, vielleicht den meisten Fällen keine Notation der Musik tradiert war. Wenn aber Bravourarien präsentiert werden sollten, musste hier Abhilfe geschaffen werden. Eine am Isthmos gefundene Ehreninschrift aus dem 2. Jh. n. Chr. für einen C. Aelius Themison zeigt schlaglichtartig eine solche Abhilfe. ${ }^{95}$ Denn The-

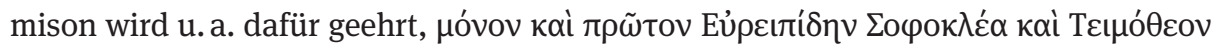
$\dot{\varepsilon} \alpha u \tau \tilde{\omega} \iota \mu \varepsilon \lambda о \pi о п ́ \sigma \alpha \nu \tau \alpha$, d.h. dass er eigene Melodien für Euripides, Sophokles und Timotheos geschaffen habe. ${ }^{96}$

Ein zweiter Nebeneffekt lag - möglicherweise - darin, dass die Dramen- und besonders Euripides-Texte mit Blick auf diese Rezitationspraxis in speziellen Auswahlen gesammelt und tradiert wurden. Es scheint mir durchaus möglich, dass der merkwürdige PHib. 179 (aus dem 3. Jh. n.Chr.) nicht etwa Zeugnis für eine uns unbekannte Version des euripideischen Herakles ist, sondern eine (stark zerstörte) Sammlung von Partien aus mehreren Stücken bietet, nicht anders als der sog. Straßburger Tragödien-Lieder-Papyrus. ${ }^{97}$

Die konzertante Aufführung bedeutet, dass die Bühnenhandlung eines Dramas als sichtbare Vermittlung eines Stoffes entfällt. Es ist eine merkwürdige Koinzidenz der Wirkungsgeschichte des antiken Dramas, dass der Siegeszug der Rezitation einhergeht mit einem Anwachsen der Bedeutung pantomimischer Darstellung in der Kaiserzeit. Man hat sogar pointiert behauptet, „dass in den euripideischen Solotänzen, etwa Phoen. 316. Or. 982. Bakch.1168. Hek. 934, der Keim zum späteren griechischrömischen Pantomimus lag."98

Auch wenn dies im Einzelnen nicht leicht zu erweisen ist, so wird doch in verschiedenen Hinsichten deutlich, dass die euripideischen Dramen ein besonders geeignetes Reservoir darstellten, aus dem der Pantomimus schöpfen konnte. Es ist sicher kein Zufall, dass Libanios in seiner Rede über die Tänzer (or. 64, 70 - 72 Foerster) Euripides neben Homer, Aischylos, Sophokles und Menander als zentralen Motivge-

93 Vgl. auch Cassius Dio 63,9,4.

94 Siehe insgesamt Heldmann 2000, 198-200.

95 Ob die Ehrung für den Flötenspieler Satyros von Samos in Delphi (SIG ${ }^{3}$ 648) auf dasselbe Problem verweist, wäre zu prüfen. Vielleicht gehört auch der Leidener Papyrus inv. 510 (3. Jh. v. Chr.) hierher, der mit Notenresten IA 1499-1509 und 784-792 (in dieser Reihenfolge!) enthält. Siehe dazu West 1992, 278 (mit weiterer Literatur).

96 TrGF 1, DID B 15, siehe dazu Heldmann 2000, 200 und insbesondere Latte 1954/1968.

97 Hierzu Hose 2008.

98 Wüst 1949, 839. 
ber für pantomimische Darstellung nennt. Ferner weisen gerade inschriftliche Zeugnisse im lateinischen Westen auf Euripides-Adaptationen in dieser Kunstform. Zwei Zeugnisse des 2. Jh. n. Chr. sind hier besonders aufschlussreich:

Der Grabstein des Pantomimen Pylades in Mailand (CIL 5, 5889) zeigt Abbildungen, die sich evident auf (Euripides') Troades und Ion beziehen. ${ }^{99}$

Eine Ehreninschrift für den Pantomimen Apolaustus in Tibur (InsIt IV, 12, Nr. 254) ${ }^{100}$ weist diesen, Lucius Aurelius Augg(ustorum) lib(ertus) Apolaustus Memphius, als ehemaligen kaiserlichen Sklaven aus, der von Lucius Verus und Marcus Aurelius freigelassen wurde, und u. a. Ehrenmitglied des Stadtrates war; im Oberteil der Ehrenbasis sind sechs Siegerkronen dargestellt. Zudem wird in griechischer Schrift an seine Glanzrollen erinnert: Orestes, Herakles, Tympanistai, Bakchen, Hippolytos. Mit Ausnahme der sophokleischen Tympanistai sind damit in der Hauptsache Dramen des Euripides genannt, die Apolaustus ohne Worte tanzte.

Resümee: Betrachtet man die Rezeptionsgeschichte der euripideischen Dramen nach dem Tod des Dichters in der Antike, so lässt sich ein Differenzierungs- und Spezialisierungsprozess erkennen, der die griechische Dramatik insgesamt kennzeichnet, an dem aber die euripideische Tragödie wohl besonderen Anteil hat: Ausgehend vom Gesamtkunstwerk des 5. Jh. v.Chr. über die Wiederaufführung dieses Gesamtkunstwerks als Bühnenerlebnis im 4. Jh. v.Chr. führt der Weg einerseits zur Rezeption als Text, die erstmals Aristoteles' Poetik programmatisch formuliert, andererseits zur konzertanten Rezitation, d.h. zu Worten ohne Handlung, wie auch zur Pantomime, zur Handlung ohne Worte. In der Kaiserzeit gibt es mithin drei Formen der euripideischen Tragödie: den Text, die Rezitation und die stumme Darstellung. Das alte Drama tritt dagegen in den Hintergrund.

\section{Literatur}

Abgekürzt werden zitiert:

SIG³: Sylloge inscriptionum Graecarum, a Guilelmo Dittenbergero condita et aucta, Vol. 2, Leipzig 31917, Vol. 3, Leipzig ${ }^{3} 1920$.

TrGF 1: Tragicorum Graecorum Fragmenta, Vol. 1, Didascaliae Tragicae, Catalogi Tragicorum et Tragoediarum, Testimonia et Fragmenta Tragicorum Minorum, ed. Bruno Snell, Göttingen 21986.

TrGF 2: Tragicorum Graecorum Fragmenta, Vol. 2, Fragmenta Adespota, edd. Richard Kannicht et Bruno Snell, Göttingen 1981.

TrGF 5: Tragicorum Graecorum Fragmenta, Vol. 5, Euripides, ed. Richard Kannicht (2 Tle.), Göttingen 2004.

99 Blänsdorf 2004, 106 u. 112; Fugmann 1988, 18-23.

100 Siehe dazu Herz 1990, $179 f$. 
Bekker 1814: Immanuel Bekker (Hg.), Anecdota Graeca, Berlin (ND Graz 1965).

Blänsdorf 1990: Jürgen Blänsdorf (Hg.), Theater und Gesellschaft im Imperium Romanum. Théatre et société dans l'empire romain, Tübingen.

Blänsdorf 2004: Jürgen Blänsdorf, „Das römische Theaterwesen der Kaiserzeit im Spiegel der Inschriften“, in: Joachim Fugmann, Markus Janka, Ulrich Schmitzer u. Helmut Seng (Hgg.), Theater, Theaterpraxis, Theaterkritik im kaiserzeitlichen Rom, München/Leipzig, 103-131.

Blass 1898: Friedrich Blass, Die attische Beredsamkeit. Dritte Abteilung. Zweiter Abschnitt: Demosthenes' Genossen und Gegner, '2Leipzig.

Bosher 2012: Kathryn Bosher (Hg.), Theater Outside Athens. Drama in Greek Sicily and South Italy, Cambridge.

Brüggemann 2011: Thomas Brüggemann, „Überlegungen zum Theater im Hellenismus“, Archiv für Papyrusforschung 57, 195-220.

Burkert 1975: Walter Burkert, „Aristoteles im Theater. Zur Datierung des 3. Buchs der 'Rhetorik' und der 'Poetik'“, Museum Helveticum 32, 67-72.

Colomo 2011: Daniela Colomo, „Euripides’ Ur-Medea between Hypothesis and Declamation“, Zeitschrift für Papyrologie und Epigraphike 176, 45-51.

Csapo u. a. 2014: Eric Csapo, Hans Rupprecht Goette, John Richard Green u. Peter Wilson (Hgg.), Greek Theatre in the Fourth Century BC, Berlin/Boston.

Csapo 2010: Eric Csapo, Actors and Icons of the Ancient Theater, Malden/Oxford.

Crusius 1902: Otto Crusius, „Die Anagnostikoi (Exkurs zu Aristot. Rhet. III 12.)“, in: Festschrift Theodor Gomperz, Wien 381-387.

Deufert 2002: Marcus Deufert, Textgeschichte und Rezeption der plautinischen Komödien im Altertum, Berlin/New York.

Diggle 1998: James Diggle (Hg.), Tragicorum Graecorum Fragmenta Selecta, Oxford.

Dilts 1992: Mervin R. Dilts (Hg.), Scholia in Aeschinem, Stuttgart/Leipzig.

Easterling u. Hall 2002: Pat E. Easterling u. Edith Hall (Hgg.), Greek and Roman Actors. Aspects of an Ancient Profession, Cambridge.

Easterling 1994: Pat E. Easterling, „Euripides outside Athens: A speculative note“, Illinois Classical Studies 19, 73-80.

Emonds 1941: Hilarius Emonds, Zweite Auflage im Altertum, Leipzig.

Engels 2008: Johannes Engels, Lykurg, Rede gegen Leokrates. Herausgegeben, eingeleitet und übersetzt, Darmstadt.

Erbse 1992: Hartmut Erbse, „Medeias Abschied von den Kindern (zu Eur. Med. 1078-1080)“, Hermes 120, 26- 43.

Falkner 2002: Thomas Falkner, „Scholars versus actors: text and performance in the Greek tragic scholia“, in: Easterling u. Hall 2002, 342-361.

Fraenkel 1960: Eduard Fraenkel, „Ein versteckter Tragödienvers?“, Philologus 104, 138-140.

Free 2015: Alexander Free, Geschichtsschreibung als Paideia. Lukians Schrift „Wie man Geschichte schreiben soll“ in der Bildungskultur des 2. Jh. n. Chr., München 2015.

Friedrich 1939: Wolf-Hartmut Friedrich, „Prolegomena zu den Phönissen“, Hermes 74, 265-300.

Fugmann 1988: Joachim Fugmann, Römisches Theater in der Provinz, Aalen (Schriften des Limesmuseums Aalen 41).

Fuhrmann 1994: Manfred Fuhrmann, Aristoteles, Poetik. Griechisch/Deutsch, ${ }^{2}$ Stuttgart.

Funke 1965/66: Hermann Funke, „Euripides“, Jahrbuch für Antike und Christentum 8/9, 232 - 279.

Gauly u. a. 1991: Bardo Gauly, Lutz Käppel, Rainer Klimek-Winter, Helmut Krasser, Karl-Heinz Stanzel u. Volker Uhrmeister (Hgg.), Musa Tragica. Die griechische Tragödie von Thespis bis Ezechiel. Ausgewählte Zeugnisse und Fragmente griechisch u. deutsch, Göttingen.

Harder 1985: Annette Harder, Euripides' Kresphontes and Archelaos. Introduction, Text and Commentary, Leiden. 
Heldmann 2000: Georg Heldmann, „Die griechische und lateinische Tragödie und Komödie in der Kaiserzeit“, Würzburger Jahrbücher für die Altertumswissenschaft 24, 185-205.

Herz 1990: Peter Herz, „Die musische Agonistik und der Kunstbetrieb der Kaiserzeit“, in: Blänsdorf 1990, 175-195.

Herzog 1901: Rudolf Herzog, „Ein Athlet als Schauspieler“, Philologus 60, 440-445.

v. Hesberg 2009: Henner v. Hesberg, „Hellenistische Theater - Zur Funktionalität der Räume und ihrer Bedeutung für die Polis“, in: Albrecht Matthaei u. Martin Zimmermann (Hgg.), Stadtbilder im Hellenismus, Berlin, 276-303.

Homeyer 1965: Helene Homeyer, Lukian. Wie man Geschichte schreiben soll. Griechisch und Deutsch. Hg., übers. u. erläutert, München.

Hose 1998: Martin Hose, „Fragment und Kontext. Zwei Methoden der Interpretation in der griechischen Literatur“, in: Jens Holzhausen (Hg.), $\psi u x \eta ́$ - Seele - anima. Festschrift für Karin Alt, Stuttgart/Berlin, 89-112.

Hose 2008: Martin Hose, „'Der Leser schneide dem Lied Länge ab‘. Vom Umgang mit Poesie im Hellenismus“, Hermes 136, 293-307.

Hunter 2009: Richard Hunter, Critical Moments in Classical Literature, Cambridge.

Hutchinson 1985: Gregory O. Hutchinson, Aeschylus, Septem contra Thebas. Edited with Introduction and Commentary, Oxford.

Kannicht 1996: Richard Kannicht, „Zum Corpus Euripideum“, in: AHNAIKA. Festschrift für Carl Werner Müller, Stuttgart/Leipzig, 21-31.

Kassel 1965: Rudolf Kassel (Hg.), Aristotelis De arte poetica liber, Oxford.

Kassel 1976: Rudolf Kassel (Hg.), Aristotelis Ars Rhetorica, Berlin/New York.

Latte 1954/1968: Kurt Latte, „Zur Geschichte der griechischen Tragödie in der Kaiserzeit“, in: Kurt Latte, Kleine Schriften zu Religion, Recht, Literatur und Sprache der Griechen und Römer, München, 590-592.

Lauriola u. Demetriou 2015: Rosanna Lauriola u. Kyriakos N. Demetriou (Hgg.), Brill's Companion to the Reception of Euripides, Leiden.

Le Guen 1995: Brigitte Le Guen, „Théatre et cités à l' époque hellénistque: mort de la cité“ - 'mort du théatre'? “, REG 108, 59-90.

Luppe 2010: Wolfgang Luppe, „Ein weiteres Zeugnis für zwei MHDEIA-Dramen des Euripides“, Zeitschrift für Papyrologie und Epigraphik 173, 15-16.

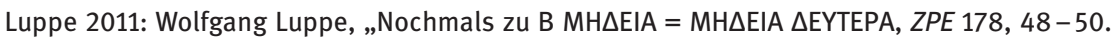

Mastronarde 1994: Donald J. Mastronarde, Euripides, Phoenissae. Ed. with Introd. and Comm., Cambridge.

Matthiessen 1994: Kjeld Matthiessen, Rez. zu „James Diggle, The Textual Transmission of Euripides' Orestes, Oxford 1991, Euripides, Phoenissae, edited by Donald J. Mastronarde, Leipzig 1988“, Gnomon 66, 661-667.

Mehl 2011: Andreas Mehl, „Mord im Theater: Euripides’ zwei ,Medeen“ und einige Folgerungen“, Archiv für Papyrusforschung 57, 274-288.

Mette 1977: Hans Joachim Mette, Urkunden dramatischer Aufführungen in Griechenland, Berlin/New York 1977.

Millis u. Olson 2012: Benjamin W. Millis u. S. Douglas Olson, Inscriptional Records for the Dramatic Festivals in Athens. IG II/2318-2325 and Related Texts, Leiden/Boston.

Moretti 1953: Luigi Moretti, Iscrizioni agonistiche greche, Rom.

Mülke 2008: Markus Mülke, Der Autor und sein Text. Die Verfälschung des Originals im Urteil antiker Autoren, Berlin/New York.

Müller 1996/1999: „Die thebanische Trilogie des Sophokles und ihre Aufführung im Jahre 401“, in: Müller 1999, 215-248 (zuerst 1996).

Müller 1999: Carl Werner Müller, Kleine Schriften zur antiken Literatur und Geistesgeschichte, Stuttgart/Leipzig. 
Nervegna 2014: Sebastiana Nervegna, „Performing Classics: The Tragic Canon in the Fourth Century and Beyond“, in: Eric Csapo, Hans Rupprecht Goette, J. Richard Green u. Peter Wilson (Hgg.), Greek Theatre in the Fourth Century BC, Berlin/Boston, 157-188.

Page 1934: Denys Lionel Page, Actors' Interpolations in Greek Tragedy, Studies with Special Reference to Euripides' Iphigenia in Aulis, Oxford.

Paulsen 1999: Thomas Paulsen, Die Parapresbeia-Reden des Demosthenes und des Aischines, Trier. Perlman 1964: Shalom Perlman, „Quotations from Poetry in Attic Orators of the 4th Cent. B.C.“, American Journal of Philology 85, 155-172.

Porter 1994: John R. Porter, Studies in Euripides' Orestes, Leiden.

Rapp 2002: Christof Rapp, Aristoteles. Rhetorik, übersetzt u. erläutert, Berlin.

Robert 1897: Carl Robert, „Zur Theaterfrage“, Hermes 32, 420-453.

Rösler 1990: Wolfgang Rösler, „Die Frage der Echtheit von Sophokles’ Antigone 904-920 und die politische Funktion der attischen Tragödie“, in: Alan Sommerstein u.a. (Hgg.), Tragedy, Comedy and the Polis, Bari 1990, 81-99.

Roueché 1993: Ch. Roueché, Performers and Partisans at Aphrodisias in the Roman and Late Roman Periods, London.

Sansone 1996: David Sansone, „Plato and Euripides“, Illinois Classical Studies 21, 35-67.

Schäfer 1885: Arnold Schäfer, Demosthenes und seine Zeit, 1. Bd., 'Leipzig.

Schmid 1940: Wilhelm Schmid, Geschichte der griechischen Literatur, Erster Teil, Dritter Band, Die griechische Literatur zur Zeit der attischen Hegemonie nach dem Eingreifen der Sophistik. Erste Hälfte, München.

Schöpsdau 1977: Platon. Werke in acht Bänden. Hg. v. Gunther Eigler, Achter Band, zweiter Teil. Gesetze Buch VII-XII, bearbeitet von Klaus Schöpsdau, Darmstadt.

Schwartz 1887: Eduard Schwartz (collegit, recensuit, edidit), Scholia in Euripidem, Vol. I, Berlin.

Seeck 1979: Gustav Adolf Seeck (Hg.), Das griechische Drama, Darmstadt.

Seeck 1979a: Gustav Adolf Seeck, „Geschichte der griechischen Tragödie“, in: Seeck 1979, $155-203$.

Seeck 1990: Gustav Adolf Seeck, „Lukian und die griechische Tragödie“, in: Blänsdorf 1990, $233-241$.

Seidensticker 1982: Bernd Seidensticker, Palintonos Harmonia. Studien zu komischen Elementen in der griechischen Tragödie, Göttingen.

Solmsen 1931: Friedrich Solmsen, Antiphonstudien, Berlin.

Taplin 2012: Oliver Taplin, „How was Athenian tragedy played in the Greek West?“, in: Kathryn Bosher (Hg.), Theater Outside Athens. Drama in Greek Sicily and South Italy, Cambridge, $226-250$.

Tedeschi 2002: Gennaro Tedeschi, „Lo spettacolo in età ellenistica e tardo antica nelle documentazioni epigrafica e papiracea“, Papyrologia Lupiensia 11, 87-187.

Wagner 1995: Ulrike Wagner, „Reprisen im Athener Dionysos-Theater im 5. und 4. Jh.“, in: Egert Pöhlmann, Studien zur Bühnendichtung und zum Theaterbau in der Antike, Frankfurt/Berlin/Bern/New York 1995, 173-178.

Wankel 1976: Hermann Wankel, Demosthenes. Rede für Ktesiphon über den Kranz. Erläutert und mit einer Einleitung versehen, 2 Halbbände, Heidelberg.

Webster 1963: Thomas B. L. Webster, Griechische Bühnenaltertümer, Göttingen.

Welcker 1841: Friedrich Gottlieb Welcker, Die griechischen Tragödien mit Rücksicht auf den epischen Cyclus geordnet, 3. Abtheilung, Bonn.

West 1992: Martin L. West, Ancient Greek Music, Oxford.

Wilamowitz 1880/1935: Ulrich v. Wilamowitz-Moellendorff, „Exkurse zu Euripides Medea“, in: ders, Kleine Schriften, Bd. 1, Berlin 17-59 (zuerst 1880).

Wüst 1949: Ernst Wüst, „Pantomimus“, RE XVIII,3, 833-869. 
Xanthakis-Karamanos 1980: Georgia Xanthakis-Karamanos, Studies in Fourth-Century Tragedy, Athen.

Zetzel 2010: James E. G. Zetzel, Rez. zu Mülke 2008, Gnomon 82, 273-275.

Ziegler u. Gärtner 1994: Konrat Ziegler (rec.), Plutarchi Vitae Parallelae, Vol. I, Fasc. 2, ed. corr. cum add. cur. Hans Gärtner, Stuttgart/Leipzig.

Ziegler 1955: Konrat Ziegler (übers.), Plutarch, Große Griechen und Römer, Bd. 2, Zürich/München. 